Bull. Korean Math. Soc. 51 (2014), No. 5, pp. 1299-1324

http://dx.doi.org/10.4134/BKMS.2014.51.5.1299

\title{
UNIFORM ATTRACTORS FOR NON-AUTONOMOUS NONCLASSICAL DIFFUSION EQUATIONS ON $\mathbb{R}^{N}$
}

\author{
Cung The Anh and Nguyen Duong Toan
}

\begin{abstract}
We prove the existence of uniform attractors $\mathcal{A}_{\varepsilon}$ in the space $H^{1}\left(\mathbb{R}^{N}\right) \cap L^{p}\left(\mathbb{R}^{N}\right)$ for the following non-autonomous nonclassical diffusion equations on $\mathbb{R}^{N}$,

$$
u_{t}-\varepsilon \Delta u_{t}-\Delta u+f(x, u)+\lambda u=g(x, t), \varepsilon \in(0,1] .
$$
\end{abstract}

The upper semicontinuity of the uniform attractors $\left\{\mathcal{A}_{\varepsilon}\right\}_{\varepsilon \in[0,1]}$ at $\varepsilon=0$ is also studied.

\section{Introduction}

In this paper we consider the following non-autonomous equation

$$
\left\{\begin{array}{l}
u_{t}-\varepsilon \Delta u_{t}-\Delta u+f(x, u)+\lambda u=g(t, x), x \in \mathbb{R}^{N}, t>\tau, \\
\left.u\right|_{t=\tau}=u_{\tau},
\end{array}\right.
$$

where $\varepsilon \in[0,1]$, the nonlinearity $f$ and the external force $g$ satisfy some certain conditions specified later. This equation is known as the nonclassical diffusion equation when $\varepsilon>0$, and the reaction-diffusion equation when $\varepsilon=0$.

Nonclassical diffusion equation arises as a model to describe physical phenomena, such as non-Newtonian flows, soil mechanic, and heat conduction (see, e.g., $[1,7,13,14]$ ). The long-time behavior of solutions to problem (1.1) has been studied extensively in recent years, for both autonomous case $[10,11,16,18]$ and non-autonomous case $[2,3,11]$. However, to the best of our knowledge, most existing results related to the problem are valid in bounded domains, except the recent work [3] where the existence of pullback attractors of the problem (1.1) on $\mathbb{R}^{N}$ was proved. In this paper we will study the existence and upper semicontinuity of uniform attractors of a family of processes associated to problem (1.1) in the case of unbounded domains, the nonlinearity of polynomial type, and the external force $g$ depending on time $t$. To

Received April 4, 2012; Revised May 23, 2014.

2010 Mathematics Subject Classification. 35B41, 35K70, 35D30.

Key words and phrases. nonclassical diffusion equation, uniform attractor, unbounded domain, upper semicontinuity, tail estimates method, asymptotic a priori estimate method.

This work is supported by the Vietnam Ministry of Education and Training under grant number B2013-17-42. 
study the existence of weak solutions to problem (1.1), we assume the following conditions:

(H1) The continuous nonlinearity $f(x, u)$ satisfies

$$
\begin{gathered}
f(x, u) u \geq \alpha_{1}|u|^{p}-\beta_{1}(x), \\
|f(x, u)| \leq \alpha_{2}|u|^{p-1}+\beta_{2}(x),
\end{gathered}
$$

for some $p \geq 2$, where $\alpha_{1}, \alpha_{2}>0, \beta_{1} \in L^{1}\left(\mathbb{R}^{N}\right)$ and $\beta_{2} \in L^{\frac{p}{p-1}}\left(\mathbb{R}^{N}\right)$ are two nonnegative functions. Moreover, we assume that

$$
f_{u}^{\prime}(x, u) \geq-\ell
$$

where $\ell$ is a positive constant. For $F(x, u)=\int_{0}^{u} f(x, \tau) d \tau$, we assume that

$$
\alpha_{3}|u|^{p}-\beta_{3}(x) \leq F(x, u) \leq \alpha_{4}|u|^{p}+\beta_{4}(x),
$$

where $\alpha_{3}, \alpha_{3}>0$ and $\beta_{3}, \beta_{4} \in L^{1}\left(\mathbb{R}^{N}\right)$ are nonnegative functions.

(H2) The external force $g \in L_{b}^{2}\left(\mathbb{R} ; L^{2}\left(\mathbb{R}^{N}\right)\right)$, that is, $g \in L_{\text {loc }}^{2}\left(\mathbb{R} ; L^{2}\left(\mathbb{R}^{N}\right)\right)$ satisfying

$$
\sup _{t \in \mathbb{R}} \int_{t}^{t+1}\|g(s)\|_{L^{2}\left(\mathbb{R}^{N}\right)}^{2} d s<+\infty .
$$

The main aim of this paper is to prove the existence of uniform attractors $\mathcal{A}_{\varepsilon}, \varepsilon \in[0,1]$, for problem (1.1) on the whole space $\mathbb{R}^{N}$ and to prove the upper semicontinuity of $\left\{\mathcal{A}_{\varepsilon}\right\}_{\varepsilon \in[0,1]}$ at $\varepsilon=0$. As we know, there are two main difficulties in studying problem (1.1) on $\mathbb{R}^{N}$ in the case $\varepsilon>0$. The first one is the unboundedness of the domain $\mathbb{R}^{N}$, this makes Sobolev embeddings are no longer compact. The second one is the appearance of the term $-\varepsilon \Delta u_{t}$, then if the initial datum $u_{\tau}$ belongs to $H^{1}\left(\mathbb{R}^{N}\right) \cap L^{p}\left(\mathbb{R}^{N}\right)$, the solution with initial condition $u(\tau)=u_{\tau}$ is always in $H^{1}\left(\mathbb{R}^{N}\right) \cap L^{p}\left(\mathbb{R}^{N}\right)$ and has no higher regularity, which is similar to hyperbolic equations. These bring some essential difficulties in proving the existence of solutions and existence of uniform attractors. On the other hand, since uniform attractors are not "invariant" like global attractors, it introduces some significant difficulty when one wants to show the upper semicontinuity of a family of uniform attractors $\left\{\mathcal{A}_{\varepsilon}\right\}_{\varepsilon \in[0,1]}$ with respect to the parameter $\varepsilon$.

Let us describe the method used in this paper. First, the existence of a unique weak solution is proved by the Galerkin approximation and the compactness method. Then, we show the existence of uniform attractors in various spaces under some stronger conditions of the external force $g$, namely conditions (H2') and (H2"), which were given in Section 3. Under the condition (H2') of $g$, using the so-called "tail estimates" method, which was introduced by B. Wang in [15], we first prove the existence of an $\left(H^{1}\left(\mathbb{R}^{N}\right) \cap\right.$ $\left.L^{p}\left(\mathbb{R}^{N}\right), L^{2}\left(\mathbb{R}^{N}\right)\right)$-uniform attractor. Next, under condition (H2") of $g$, using the asymptotic a priori estimate method in [9], we prove the asymptotic compactness in $L^{p}\left(\mathbb{R}^{N}\right)$ of the corresponding process, and thus obtain 
the existence of an $\left(H^{1}\left(\mathbb{R}^{N}\right) \cap L^{p}\left(\mathbb{R}^{N}\right), L^{p}\left(\mathbb{R}^{N}\right)\right)$-uniform attractor. After that, by verifying Condition (C) introduced in [8] we get the existence of an $\left(H^{1}\left(\mathbb{R}^{N}\right) \cap L^{p}\left(\mathbb{R}^{N}\right), H^{1}\left(\mathbb{R}^{N}\right) \cap L^{p}\left(\mathbb{R}^{N}\right)\right)$-uniform attractor. Finally, by using the structure of uniform attractors, that is, a uniform attractor can be viewed as a union of kernel sections (see Definition 2.4), and the continuous dependence of solutions to problem (1.1) on $\varepsilon$ as $\varepsilon \rightarrow 0^{+}$established in Lemma 5.1, we prove the upper semicontinuity of the family of uniform attractors $\left\{\mathcal{A}_{\varepsilon}\right\}_{\varepsilon \in[0,1]}$ at $\varepsilon=0$.

The paper is organized as follows. In Section 2, for convenience of the reader, we recall some results on uniform attractors and the space of translation bounded functions. Section 3 proves the existence and weak continuity of a family of processes associated to the problem. In Section 4 , we prove the existence of uniform attractors $\mathcal{A}_{\varepsilon}$ for the family of processes in various spaces. The upper semicontinuity of uniform attractors $\left\{\mathcal{A}_{\varepsilon}\right\}_{\varepsilon \in[0,1]}$ at $\varepsilon=0$ is investigated in Section 5. In the last section, we give some relationships between the above uniform attractor, the pullback attractor obtained in [3], and the global attractor formally obtained when the external force is time-independent.

Throughout this paper, we denote by $\|\cdot\|,(\cdot, \cdot)$ the norm and scalar product in $L^{2}\left(\mathbb{R}^{N}\right)$, respectively. We denote by $C$ an arbitrary constant, which is different from line to line, even in a same line.

\section{Preliminaries}

\subsection{Uniform attractors}

Let $\Sigma$ be a parameter set, $X, Y$ be two Banach spaces. A family $\left\{U_{\sigma}(t, \tau)\right.$, $t \geq \tau, \tau \in \mathbb{R}\}, \sigma \in \Sigma$, is said to be a family of processes from $X$ to $Y$ if for each $\sigma \in \Sigma,\left\{U_{\sigma}(t, \tau)\right\}$ is a process, that is, the two-parameter family of mappings $\left\{U_{\sigma}(t, \tau)\right\}$ from $X$ to $Y$ satisfies

$$
\begin{aligned}
U_{\sigma}(t, s) U_{\sigma}(s, \tau) & =U_{\sigma}(t, \tau), \forall t \geq s \geq \tau, \tau \in \mathbb{R}, \\
U_{\sigma}(\tau, \tau) & =I d, \text { the identity operator, } \tau \in \mathbb{R},
\end{aligned}
$$

where $\Sigma$ is called the symbol space, $\sigma \in \Sigma$ is the symbol. Denote by $\mathcal{B}(X)$ the set of all bounded subsets of $X$.

Definition 2.1. A set $B_{0} \in \mathcal{B}(Y)$ is said to be an $(X, Y)$-uniform (w.r.t. $\sigma \in \Sigma)$ absorbing set for $\left\{U_{\sigma}(t, \tau)\right\}_{\sigma \in \Sigma}$, if for any $\tau \in \mathbb{R}$, and $B \in \mathcal{B}(X)$, there exists $T_{0} \geq \tau$ such that $\bigcup_{\sigma \in \Sigma} U_{\sigma}(t, \tau) B \subset B_{0}$ for all $t \geq T_{0}$.

Definition 2.2. A family of processes $\left\{U_{\sigma}(t, \tau)\right\}_{\sigma \in \Sigma}$ is called $(X, Y)$-uniformly (w.r.t. $\sigma \in \Sigma$ ) asymptotically compact if for any $\tau \in \mathbb{R}$, any $B \in \mathcal{B}(X)$, we have $\left\{U_{\sigma_{n}}\left(t_{n}, \tau\right) x_{n}\right\}$ is relatively compact in $Y$, where $\left\{x_{n}\right\} \subset B,\left\{t_{n}\right\} \subset[\tau,+\infty)$, $t_{n} \rightarrow+\infty$ and $\left\{\sigma_{n}\right\} \subset \Sigma$ are arbitrary.

Definition 2.3. A subset $\mathcal{A}_{\Sigma} \subset Y$ is said to be an $(X, Y)$-uniform attractor of the family of processes $\left\{U_{\sigma}(t, \tau)\right\}_{\sigma \in \Sigma}$ if 
(1) $\mathcal{A}_{\Sigma}$ is compact in $Y$;

(2) for an arbitrary fixed $\tau \in \mathbb{R}$ and $B \in \mathcal{B}(X)$ we have

$$
\lim _{t \rightarrow \infty}\left(\sup _{\sigma \in \Sigma}\left(\operatorname{dist}_{Y}\left(U_{\sigma}(t, \tau) B, \mathcal{A}_{\Sigma}\right)\right)=0,\right.
$$

where $\operatorname{dist}_{E}(\cdot, \cdot)$ denotes the Hausdorff semidistance in a Banach space E

$$
\operatorname{dist}_{E}(A, B)=\sup _{x \in A} \inf _{y \in B}\|x-y\|_{E}
$$

(3) if $\mathcal{A}_{\Sigma}^{\prime}$ is a closed subset of $Y$ satisfying (2), then $\mathcal{A}_{\Sigma} \subset \mathcal{A}^{\prime}{ }_{\Sigma}$.

Definition 2.4. The kernel $\mathcal{K}$ of a process $\{U(t, \tau)\}$ acting on $X$ consists of all bounded complete trajectories of the process $\{U(t, \tau)\}$ :

$$
\mathcal{K}=\left\{u(\cdot) \mid U(t, \tau) u(\tau)=u(t), \operatorname{dist}(u(t), u(0)) \leq C_{u}, \forall t \geq \tau, \tau \in \mathbb{R}\right\} .
$$

For $s \in \mathbb{R}$, the set $\mathcal{K}(s)=\{u(s) \mid u(\cdot) \in \mathcal{K}\}$ is said to be kernel section at time $s$.

We will use the following result on the existence and structure of bi-spaces uniform attractors.

Theorem 2.1 ([4]). Assume that the family of processes $\left\{U_{\sigma}(t, \tau)\right\}_{\sigma \in \Sigma}$ satisfies the following conditions:

(1) $\Sigma$ is weakly compact, and $\left\{U_{\sigma}(t, \tau)\right\}_{\sigma \in \Sigma}$ is $(X \times \Sigma, Y)$-weakly continuous, that is, for any fixed $t \geq \tau$, the mapping $(u, \sigma) \mapsto U_{\sigma}(t, \tau) u$ is weakly continuous in $Y$. Moreover, there is a weakly continuous semigroup $\{T(h)\}_{h \geq 0}$ acting on $\Sigma$ satisfying

$T(h) \Sigma=\Sigma, U_{\sigma}(t+h, \tau+h)=U_{T(h) \sigma}(t, \tau), \forall \sigma \in \Sigma, t \geq \tau, h \geq 0 ;$

(2) $\left\{U_{\sigma}(t, \tau)\right\}_{\sigma \in \Sigma}$ has an $(X, Y)$-uniform (w.r.t. $\sigma \in \Sigma$ ) absorbing set $B_{0}$;

(3) $\left\{U_{\sigma}(t, \tau)\right\}_{\sigma \in \Sigma}$ is $(X, Y)$-uniformly (w.r.t. $\sigma \in \Sigma$ ) asymptotically compact.

Then it possesses an $(X, Y)$-uniform attractor $\mathcal{A}_{\Sigma}$, and

$$
\mathcal{A}_{\Sigma}=\bigcup_{\sigma \in \Sigma} \mathcal{K}_{\sigma}(s), \forall s \in \mathbb{R},
$$

where $\mathcal{K}_{\sigma}(s)$ is the kernel section at time $s$ of the process $U_{\sigma}(t, \tau)$.

\subsection{The translation bounded functions}

Definition 2.5. Let $\mathcal{E}$ be a reflexive Banach space. A function $\varphi \in L_{\text {loc }}^{2}(\mathbb{R} ; \mathcal{E})$ is said to be translation bounded if

$$
\|\varphi\|_{b}^{2}=\|\varphi\|_{L_{b}^{2}(\mathbb{R} ; \mathcal{E})}^{2}=\sup _{t \in \mathbb{R}} \int_{t}^{t+1}\|\varphi\|_{\mathcal{E}}^{2} d s<\infty .
$$

For $g \in L_{b}^{2}\left(\mathbb{R} ; L^{2}\left(\mathbb{R}^{N}\right)\right)$, we denote by $\mathcal{H}_{w}(g)$ the closure of the set $\{g(\cdot+$ $h) \mid h \in \mathbb{R}\}$ in the space $L_{b}^{2}\left(\mathbb{R} ; L^{2}\left(\mathbb{R}^{N}\right)\right)$ with the weak topology. The following results were proved in [5]. 
Lemma 2.2 ([5, Chapter 5, Proposition 4.2]).

(1) For all $\sigma \in \mathcal{H}_{w}(g),\|\sigma\|_{b}^{2} \leq\|g\|_{b}^{2}$;

(2) The translation group $\{T(h)\}$ is weakly continuous on $\mathcal{H}_{w}(g)$;

(3) $T(h) \mathcal{H}_{w}(g)=\mathcal{H}_{w}(g)$ for $h \geq 0$;

(4) $\mathcal{H}_{w}(g)$ is weakly compact.

\section{Existence and weak continuity of the family of associated processes}

Definition 3.1. A function $u(t, x)$ is called a weak solution of problem (1.1) on the interval $[\tau, T]$ if

$$
\begin{gathered}
u \in C\left([\tau, T] ; H^{1}\left(\mathbb{R}^{N}\right)\right) \cap L^{p}\left(\tau, T ; L^{p}\left(\mathbb{R}^{N}\right)\right), u_{t} \in L^{2}\left(\tau, T ; H^{1}\left(\mathbb{R}^{N}\right)\right), \\
u(x, \tau)=u_{\tau}(x) \text { for a.e. } x \in \mathbb{R}^{N},
\end{gathered}
$$

and

$$
\begin{aligned}
& \int_{\tau}^{T} \int_{\mathbb{R}^{N}} u_{t} \varphi+\varepsilon \int_{\tau}^{T} \int_{\mathbb{R}^{N}} \nabla u_{t} \nabla \varphi+\int_{\tau}^{T} \int_{\mathbb{R}^{N}} \nabla u \nabla \varphi \\
& +\int_{\tau}^{T} \int_{\mathbb{R}^{N}} f(x, u) \varphi+\lambda \int_{\tau}^{T} \int_{\mathbb{R}^{N}} u \varphi=\int_{\tau}^{T} \int_{\mathbb{R}^{N}} g(s) \varphi
\end{aligned}
$$

for all test functions $\varphi \in C^{\infty}\left([\tau, T] \times \mathbb{R}^{N}\right)$.

Theorem 3.1. Assume that $f$ satisfies condition (H1), $g$ satisfies condition (H2). Then for any $u_{\tau} \in H^{1}\left(\mathbb{R}^{N}\right) \cap L^{p}\left(\mathbb{R}^{N}\right)$, any $\sigma \in \mathcal{H}_{w}(g)$ and any $T>\tau$, $\tau \in \mathbb{R}$ given, problem (1.1) (with $\sigma$ in place of $g$ ) has a unique weak solution $u$ on $[\tau, T]$ and the weak solution depends continuously on the initial data. Moreover, for any $t>\tau$, we have

$$
\|u(t)\|^{2}+\varepsilon\|\nabla u(t)\|^{2} \leq C\left(e^{-\delta(t-\tau)}\left(\left\|u_{\tau}\right\|^{2}+\varepsilon\left\|\nabla u_{\tau}\right\|^{2}\right)+1+\frac{1}{1-e^{-\delta}}\|g\|_{b}^{2}\right),
$$

where $\delta=\min \{1, \lambda\}$.

Proof. Although the existence of a weak solution was proved in [3], we present here another (simpler) proof for the completeness and convenience of the reader.

i) Existence. For each integer $n \geq 1$, we denote by

$$
u_{n}(t)=\sum_{j=1}^{n} \gamma_{n j}(t) w_{j}
$$

a solution of

$$
\left\{\begin{aligned}
\frac{d}{d t}\left(u_{n}, w_{j}\right)= & -\varepsilon\left(\nabla \partial_{t} u_{n}, \nabla w_{j}\right)-\left(\nabla u_{n}, \nabla w_{j}\right)-\left\langle f\left(x, u_{n}\right), w_{j}\right\rangle \\
& -\lambda\left(u_{n}, w_{j}\right)+\left(\sigma(t), w_{j}\right), \\
\left(u_{n}(\tau), w_{j}\right)= & \left(u_{\tau}, w_{j}\right), j=1, \ldots, n,
\end{aligned}\right.
$$


where $\left\{w_{j}: j \geq 1\right\} \subset H^{1}\left(\mathbb{R}^{N}\right) \cap L^{p}\left(\mathbb{R}^{N}\right)$ is a Hilbert basis of $L^{2}\left(\mathbb{R}^{N}\right)$ such that $\operatorname{span}\left\{w_{j}\right\}_{j \geq 1}$ is dense in $H^{1}\left(\mathbb{R}^{N}\right) \cap L^{p}\left(\mathbb{R}^{N}\right)$. In (3.2), replacing $w_{j}$ by $u_{n}$, we get

$$
\begin{aligned}
& \frac{1}{2} \frac{d}{d t}\left(\left\|u_{n}\right\|^{2}+\varepsilon\left\|\nabla u_{n}\right\|^{2}\right)+\left\|\nabla u_{n}\right\|^{2}+\int_{\mathbb{R}^{N}} f\left(x, u_{n}\right) u_{n} d x+\lambda\left\|u_{n}\right\|^{2} \\
= & \int_{\mathbb{R}^{N}} \sigma(t) u_{n} d x .
\end{aligned}
$$

By (1.2), we have

$$
\int_{\mathbb{R}^{N}} f\left(x, u_{n}\right) u_{n} d x \geq \alpha_{1}\left\|u_{n}\right\|_{L^{p}\left(\mathbb{R}^{N}\right)}^{p}-\left\|\beta_{1}\right\|_{L^{1}\left(\mathbb{R}^{N}\right)} .
$$

Applying the Cauchy inequality, we have

$$
\int_{\mathbb{R}^{N}} \sigma(t) u_{n} d x \leq\|\sigma(t)\|\left\|u_{n}\right\| \leq \frac{1}{2 \lambda}\|\sigma(t)\|^{2}+\frac{\lambda}{2}\left\|u_{n}\right\|^{2} .
$$

Combining (3.3)-(3.5), we get

$$
\begin{aligned}
& \frac{d}{d t}\left(\left\|u_{n}\right\|^{2}+\varepsilon\left\|\nabla u_{n}\right\|^{2}\right)+2\left\|\nabla u_{n}\right\|^{2}+2 \alpha_{1}\left\|u_{n}\right\|_{L^{p}\left(\mathbb{R}^{N}\right)}^{p} \\
\leq & \frac{1}{\lambda}\|\sigma(t)\|^{2}+2\left\|\beta_{1}\right\|_{L^{1}\left(\mathbb{R}^{N}\right)} .
\end{aligned}
$$

Integrating (3.6) from $\tau$ to $t, t \in[\tau, T]$, we have

$$
\begin{aligned}
& \left\|u_{n}(t)\right\|^{2}+\varepsilon\left\|\nabla u_{n}(t)\right\|^{2}+2 \int_{\tau}^{t}\left\|\nabla u_{n}(s)\right\|^{2} d s+2 \alpha_{1} \int_{\tau}^{t}\left\|u_{n}(s)\right\|_{L^{p}\left(\mathbb{R}^{N}\right)}^{p} d s \\
\leq & \left\|u_{n}(\tau)\right\|^{2}+\varepsilon\left\|\nabla u_{n}(\tau)\right\|^{2}+\frac{1}{\lambda} \int_{\tau}^{T}\|\sigma(s)\|^{2} d s+2(T-\tau)\left\|\beta_{1}\right\|_{L^{1}\left(\mathbb{R}^{N}\right)} .
\end{aligned}
$$

This inequality implies that

(3.7) $\quad\left\{u_{n}\right\}$ is bounded in $L^{\infty}\left(\tau, T ; H^{1}\left(\mathbb{R}^{N}\right)\right) \cap L^{p}\left(\tau, T ; L^{p}\left(\mathbb{R}^{N}\right)\right)$.

Then, there exists a subsequence of $\left\{u_{n}\right\}$ (still denoted by $\left\{u_{n}\right\}$ ) such that

$$
u_{n} \rightarrow u \text { weakly-star in } L^{\infty}\left(\tau, T ; H^{1}\left(\mathbb{R}^{N}\right)\right) .
$$

We deduce in particular from (3.7) that

$$
\Delta u_{n} \rightarrow \Delta u \text { in } L^{2}\left(\tau, T ; H^{-1}\left(\mathbb{R}^{N}\right)\right) .
$$

On the other hand, replacing $w_{j}$ by $\partial_{t} u_{n}$ in (3.2), we get

$$
\begin{aligned}
& \left\|\partial_{t} u_{n}\right\|^{2}+\varepsilon\left\|\nabla \partial_{t} u_{n}\right\|^{2}+\frac{1}{2} \frac{d}{d t}\left(\left\|\nabla u_{n}\right\|^{2}+\lambda\left\|u_{n}\right\|^{2}+2 \int_{\mathbb{R}^{N}} F\left(x, u_{n}\right) d x\right) \\
= & \int_{\mathbb{R}^{N}} \sigma(t) \partial_{t} u_{n} d x \leq \frac{1}{2}\|\sigma(t)\|^{2}+\frac{1}{2}\left\|\partial_{t} u_{n}\right\|^{2} .
\end{aligned}
$$

Integrating (3.10) from $\tau$ to $T$, we obtain

$$
\left\{\partial_{t} u_{n}\right\} \text { is bounded in } L^{2}\left(\tau, T ; H^{1}\left(\mathbb{R}^{N}\right)\right),
$$


thus

$$
\partial_{t} u_{n} \rightarrow u_{t} \text { in } L^{2}\left(\tau, T ; H^{1}\left(\mathbb{R}^{N}\right)\right)
$$

and

$$
\Delta \partial_{t} u_{n} \rightarrow \Delta u_{t} \text { in } L^{2}\left(\tau, T ; H^{-1}\left(\mathbb{R}^{N}\right)\right),
$$

up to a subsequence. Using (1.3), for $p^{\prime}=\frac{p}{p-1}$, we get

$$
\begin{aligned}
\int_{\mathbb{R}^{N}}\left|f\left(x, u_{n}\right)\right|^{p^{\prime}} d x & \leq \int_{\mathbb{R}^{N}}\left(\alpha_{2}\left|u_{n}\right|^{p-1}+\beta_{2}(x)\right)^{p^{\prime}} d x \\
& \leq C_{p^{\prime}}\left(\alpha_{2}\left\|u_{n}\right\|_{L^{p}\left(\mathbb{R}^{N}\right)}^{p}+\left\|\beta_{2}\right\|_{L^{p^{\prime}\left(\mathbb{R}^{N}\right)}}^{p^{\prime}}\right) .
\end{aligned}
$$

From (3.7) and (3.13) we find that

$$
\left\{f\left(., u_{n}\right)\right\} \text { is bounded in } L^{p^{\prime}}\left(\tau, T ; L^{p^{\prime}}\left(\mathbb{R}^{N}\right)\right),
$$

thus

$$
f\left(\cdot, u_{n}\right) \rightarrow \chi \text { in } L^{p^{\prime}}\left(\tau, T ; L^{p^{\prime}}\left(\mathbb{R}^{N}\right)\right), \text { up to a subsequence. }
$$

We will prove that $\chi=f(x, u)$. For each $m \geq 1$, we denote $B_{m}=\left\{x \in \mathbb{R}^{N}\right.$ : $|x| \leq m\}$. Let $\theta \in C^{1}([0,+\infty))$ be a function such that $0 \leq \theta \leq 1,\left.\theta\right|_{[0,1]}=1$ and $\theta(s)=0$ for all $s \geq 2$. For each $n$ and $m$ we define

$$
v_{n, m}(x, t)=\theta\left(\frac{|x|^{2}}{m^{2}}\right) u_{n}(x, t) .
$$

We obtain from (3.7) that, for all $m \geq 1$, the sequence $\left\{v_{n, m}\right\}_{n \geq 1}$ is bounded in $L^{\infty}\left(\tau, T ; H_{0}^{1}\left(B_{2 m}\right)\right) \cap L^{p}\left(\tau, T ; L^{p}\left(B_{2 m}\right)\right)$. As $B_{2 m}$ is a bounded set, the embedding $H_{0}^{1}\left(B_{2 m}\right) \hookrightarrow L^{2}\left(B_{2 m}\right)$ is compact. Then, by Theorem 13.3 and Remark 13.1 in [12] we obtain that

$$
\left\{v_{n, m}\right\} \text { is precompact in } L^{2}\left(\tau, T ; L^{2}\left(B_{2 m}\right)\right),
$$

and thus

$$
\left\{\left.u_{n}\right|_{B_{m}}\right\} \text { is precompact in } L^{2}\left(\tau, T ; L^{2}\left(B_{m}\right)\right) \text {. }
$$

By a diagonal procedure, using (3.8), we deduce that there exists a subsequence of $\left\{u_{n}\right\}$ (still denoted by $\left\{u_{n}\right\}$ ) such that

$$
u_{n} \rightarrow u \text { a.e. in } B_{m} \times(\tau,+\infty) \text { as } n \rightarrow+\infty, \forall m \geq 1 \text {. }
$$

Then, since $f$ is continuous,

$$
f\left(x, u_{n}\right) \rightarrow f(x, u) \text { a.e. in } B_{m} \times(\tau,+\infty),
$$

and $\left\{f\left(x, u_{n}\right)\right\}$ is bounded in $L^{p^{\prime}}\left(\tau, T ; L^{p^{\prime}}\left(B_{m}\right)\right)$, by Lemma 1.3 in [6, Chapter 1], we have

From (3.14)

$$
f\left(x, u_{n}\right) \rightarrow f(x, u) \text { in } L^{p^{\prime}}\left(\tau, T ; L^{p^{\prime}}\left(B_{m}\right)\right) .
$$

$$
\left.f\left(x, u_{n}\right) \rightarrow \chi\right|_{B_{m} \times(\tau, T)} \text { in } L^{p^{\prime}}\left(\tau, T ; L^{p^{\prime}}\left(B_{m}\right)\right) .
$$


Hence

$$
\chi=f(x, u) \text { a.e. in } B_{m} \times(\tau, T), \forall m \geq 1,
$$

and thus, taking into account that $\bigcup_{m=1}^{\infty} B_{m}=\mathbb{R}^{N}$, we obtain

$$
\chi=f(x, u) \text { a.e. in } \mathbb{R}^{N} \times(\tau, T) .
$$

Now, combining (3.8), (3.9), (3.11), (3.12), (3.15) and (3.16) we see that $u$ satisfies

$u_{t}-\varepsilon \Delta u_{t}-\Delta u+f(x, u)+\lambda u=\sigma(t)$ in $H^{-1}\left(\mathbb{R}^{N}\right)+L^{p^{\prime}}\left(\mathbb{R}^{N}\right)$ for a.e. $t \in[\tau, T]$.

By standard arguments, we can check that $u$ satisfies the initial condition $u(\tau)=u_{\tau}$, and this implies that $u$ is a weak solution of problem (1.1).

ii) Uniqueness and continuous dependence. We assume that $u_{1}$ and $u_{2}$ are two solutions subject to initial data $u_{1}(\tau)$ and $u_{2}(\tau)$, respectively. Denote $w=u_{1}-u_{2}$, we have

$$
w_{t}-\varepsilon \Delta w_{t}-\Delta w+f\left(x, u_{1}\right)-f\left(x, u_{2}\right)+\lambda w=0 .
$$

Taking the inner product of $(3.17)$ in $L^{2}\left(\mathbb{R}^{N}\right)$ with $w$, then using assumption (1.4), we see that

$$
\frac{d}{d t}\left(\|w\|^{2}+\varepsilon\|\nabla w\|^{2}\right)+2\|\nabla w\|^{2}+2 \lambda\|w\|^{2} \leq 2 \ell\|w\|^{2} \leq 2 \ell\left(\|w\|^{2}+\varepsilon\|\nabla w\|^{2}\right) .
$$

By the Gronwall inequality, we obtain

$$
\|w(t)\|^{2}+\varepsilon\|\nabla w(t)\|^{2} \leq e^{2 \ell(T-\tau)}\left(\|w(\tau)\|^{2}+\varepsilon\|\nabla w(\tau)\|^{2}\right) .
$$

This proves the uniqueness (when $u_{1}(\tau)=u_{2}(\tau)$ ) and the continuous dependence on the initial data of the weak solution.

iii) The a priori estimate (3.1). Multiplying (1.1) by $u(t)$ and integrating over $\mathbb{R}^{N}$, we get

$$
\begin{aligned}
& \frac{1}{2} \frac{d}{d t}\left(\|u(t)\|^{2}+\varepsilon\|\nabla u(t)\|^{2}\right)+\|\nabla u(t)\|^{2}+\lambda\|u(t)\|^{2}+\int_{\mathbb{R}^{N}} f(x, u(t)) u(t) \\
= & \int_{\mathbb{R}^{N}} \sigma(t) u(t) .
\end{aligned}
$$

Using (1.2), the Hölder and Cauchy inequalities, after a few computations, we have

$$
\begin{aligned}
& \frac{d}{d t}\left(\|u(t)\|^{2}+\varepsilon\|\nabla u(t)\|^{2}\right)+\delta\left(\|u(t)\|^{2}+\varepsilon\|\nabla u(t)\|^{2}\right) \\
& +\zeta\left(\|u(t)\|_{L^{p}\left(\mathbb{R}^{N}\right)}^{p}+\|\nabla u(t)\|^{2}\right) \\
\leq & 2\left\|\beta_{1}\right\|_{L^{1}\left(\mathbb{R}^{N}\right)}+\frac{1}{\lambda}\|\sigma(t)\|^{2}
\end{aligned}
$$


where $\zeta=\min \left\{2 \alpha_{1}, 1\right\}$. Hence, by the Gronwall inequality, we deduce that (3.18)

$$
\begin{aligned}
& \|u(t)\|^{2}+\varepsilon\|\nabla u(t)\|^{2} \\
\leq & e^{-\delta(t-\tau)}\left(\left\|u_{\tau}\right\|^{2}+\varepsilon\left\|\nabla u_{\tau}\right\|^{2}\right)+2\left\|\beta_{1}\right\|_{L^{1}\left(\mathbb{R}^{N}\right)}+\frac{1}{\lambda} \int_{\tau}^{t} e^{-\delta(t-s)}\|\sigma(s)\|^{2} d s .
\end{aligned}
$$

On the other hand, we have

$$
\begin{aligned}
& \int_{\tau}^{t} e^{-\delta(t-s)}\|\sigma(s)\|^{2} d s \\
\leq & \left(\int_{t-1}^{t} e^{-\delta(t-s)}\|\sigma(s)\|^{2} d s+\int_{t-2}^{t-1} e^{-\delta(t-s)}\|\sigma(s)\|^{2} d s+\cdots\right) \\
\leq & \left(1+e^{-\delta}+e^{-2 \delta}+\cdots\right)\|\sigma\|_{b}^{2} \leq \frac{1}{1-e^{-\delta}}\|g\|_{b}^{2},
\end{aligned}
$$

where we have used the fact that $\|\sigma\|_{b}^{2} \leq\|g\|_{b}^{2}$ for all $\sigma \in \mathcal{H}_{w}(g)$. Combining (3.18) and (3.19), we get (3.1).

Theorem 3.1 allows us to define a family of continuous processes

$$
\left\{U_{\sigma}(t, \tau)\right\}_{\sigma \in \mathcal{H}_{w}(g)}
$$

as follows

$$
U_{\sigma}(t, \tau): H^{1}\left(\mathbb{R}^{N}\right) \cap L^{p}\left(\mathbb{R}^{N}\right) \rightarrow H^{1}\left(\mathbb{R}^{N}\right) \cap L^{p}\left(\mathbb{R}^{N}\right),
$$

where $U_{\sigma}(t, \tau) u_{\tau}$ is the unique weak solution of (1.1) (with $\sigma$ in place of $g$ ) at the time $t$ with the initial datum $u_{\tau}$ at $\tau$.

We now prove the weak continuity of the family of processes

$$
\left\{U_{\sigma}(t, \tau)\right\}_{\sigma \in \mathcal{H}_{w}(g)} .
$$

Lemma 3.2. The family of processes $\left\{U_{\sigma}(t, \tau)\right\}_{\sigma \in \mathcal{H}_{w}(g)}$ associated to problem (1.1) is $\left(H^{1}\left(\mathbb{R}^{N}\right) \cap L^{p}\left(\mathbb{R}^{N}\right) \times \mathcal{H}_{w}(g), H^{1}\left(\mathbb{R}^{N}\right) \cap L^{p}\left(\mathbb{R}^{N}\right)\right)$-weakly continuous, that is, for any $u_{\tau}^{(n)} \rightarrow u_{\tau}$ in $H^{1}\left(\mathbb{R}^{N}\right) \cap L^{p}\left(\mathbb{R}^{N}\right)$ and $\sigma_{n} \rightarrow \sigma_{0}$ in $\mathcal{H}_{w}(g)$, we have

$$
U_{\sigma_{n}}(t, \tau) u_{\tau}^{(n)} \rightarrow U_{\sigma_{0}}(t, \tau) u_{\tau} \text { in } H^{1}\left(\mathbb{R}^{N}\right) \cap L^{p}\left(\mathbb{R}^{N}\right), \quad t \geq \tau .
$$

Proof. Denote $u_{n}(t)=U_{\sigma_{n}}(t, \tau) u_{\tau}^{(n)}$, we easily see that all estimates for approximate solutions in Theorem 3.1 are still valid for $u_{n}(t)$ here. Thus, there is $w(t)$ such that

$$
u_{n} \rightarrow w \text { weakly-star in } L^{\infty}\left(\tau, t ; H^{1}\left(\mathbb{R}^{N}\right)\right),
$$

$$
u_{n} \rightarrow w \text { in } L^{p}\left(\tau, t ; L^{p}\left(\mathbb{R}^{N}\right)\right)
$$

and the sequence

$$
\left\{u_{n}(s)\right\}, \tau \leq s \leq t, \text { is bounded in } H^{1}\left(\mathbb{R}^{N}\right) \cap L^{p}\left(\mathbb{R}^{N}\right) .
$$


Using the arguments as in Theorem 3.1, we can see that

$$
\partial_{t} u_{n} \rightarrow w_{t} \text { in } L^{2}\left(\tau, t ; H^{1}\left(\mathbb{R}^{N}\right)\right),
$$

and

$$
f\left(x, u_{n}\right) \rightarrow f(x, w) \text { in } L^{p^{\prime}}\left(\tau, t ; L^{p^{\prime}}\left(\mathbb{R}^{N}\right)\right) .
$$

Hence, by combining (3.20)-(3.23), we obtain that $w$ solves the problem

$$
w_{t}-\varepsilon \Delta w_{t}-\Delta w+f(x, w)+\lambda w=\sigma_{0},\left.w\right|_{t=\tau}=u_{\tau},
$$

and therefore $w=U_{\sigma_{0}}(t, \tau) u_{\tau}$ thanks to the uniqueness of weak solutions. This completes the proof.

\section{Existence of uniform attractors}

First, we prove the existence of an $\left(H^{1}\left(\mathbb{R}^{N}\right) \cap L^{p}\left(\mathbb{R}^{N}\right), H^{1}\left(\mathbb{R}^{N}\right) \cap L^{p}\left(\mathbb{R}^{N}\right)\right)$ uniform absorbing set for the family of processes $\left\{U_{\sigma}(t, \tau)\right\}_{\sigma \in \mathcal{H}_{w}(g)}$.

Proposition 4.1. The family of processes $\left\{U_{\sigma}(t, \tau)\right\}_{\sigma \in \mathcal{H}_{w}(g)}$ associated to problem (1.1) has an $\left(H^{1}\left(\mathbb{R}^{N}\right) \cap L^{p}\left(\mathbb{R}^{N}\right), H^{1}\left(\mathbb{R}^{N}\right) \cap L^{p}\left(\mathbb{R}^{N}\right)\right)$-uniform absorbing set, which is independent of $\varepsilon$.

Proof. Multiplying (1.1) by $u+u_{t}$ in $L^{2}\left(\mathbb{R}^{N}\right)$, and after some standard computations, we get

$$
\begin{aligned}
& \quad \frac{1}{2} \frac{d}{d t}\left((\lambda+1)\|u\|^{2}+(\varepsilon+1)\|\nabla u\|^{2}+2 \int_{\mathbb{R}^{N}} F(x, u) d x\right) \\
& \quad+\|\nabla u\|^{2}+\frac{1}{2}\left\|u_{t}\right\|^{2}+\varepsilon\left\|\nabla u_{t}\right\|^{2}+\int_{\mathbb{R}^{N}} f(x, u) u d x+\frac{\lambda}{2}\|u\|^{2} \\
& \leq \frac{\lambda+1}{2}\|\sigma(t)\|^{2} .
\end{aligned}
$$

Using (1.2) and (1.5), we get two positive constants $\mu$ and $C$ such that

$$
\begin{aligned}
& \|\nabla u\|^{2}+\int_{\mathbb{R}^{N}} f(x, u) u d x+\frac{\lambda}{2}\|u\|^{2} \\
\geq & \frac{\mu}{2}\left((\lambda+1)\|u\|^{2}+(\varepsilon+1)\|\nabla u\|^{2}+2 \int_{\mathbb{R}^{N}} F(x, u) d x\right)-C .
\end{aligned}
$$

Thus, combining (4.1) and (4.2) we have

$$
\frac{d}{d t} y(t)+\mu y(t) \leq(\lambda+1)\|\sigma(t)\|^{2}+C
$$

where

$$
y(t)=(\lambda+1)\|u\|^{2}+(\varepsilon+1)\|\nabla u\|^{2}+2 \int_{\mathbb{R}^{N}} F(x, u) d x .
$$


Hence, from (1.5) we obtain

$$
\begin{aligned}
y(t) & \leq e^{-\mu(t-\tau)} y(\tau)+(\lambda+1) e^{-\mu t} \int_{\tau}^{t} e^{\mu s}\|\sigma(s)\|^{2} d s+C \\
& \leq C e^{-\mu(t-\tau)}\left(\left\|u_{\tau}\right\|^{2}+\left\|\nabla u_{\tau}\right\|^{2}+\left\|u_{\tau}\right\|_{L^{p}\left(\mathbb{R}^{N}\right)}^{p}+1\right)+\frac{\lambda+1}{1-e^{-\mu}}\|g\|_{b}^{2}+C .
\end{aligned}
$$

On the other hand, using (1.5) once again we find that

$$
y(t) \geq\|u(t)\|^{2}+\|\nabla u(t)\|^{2}+2 \alpha_{3}\|u(t)\|_{L^{p}\left(\mathbb{R}^{N}\right)}^{p}-2\left\|\beta_{3}\right\|_{L^{1}\left(\mathbb{R}^{N}\right)} .
$$

Now, by combining (4.3) and (4.4), we can choose $T_{1}$ and $\rho_{0}$ such that

$$
\|u(t)\|^{2}+\|\nabla u(t)\|^{2}+\|u(t)\|_{L^{p}\left(\mathbb{R}^{N}\right)}^{p} \leq \rho_{0}
$$

for all $t \geq T_{1}, u_{\tau} \in B$ and $\sigma \in \mathcal{H}_{w}(g)$. This completes the proof.

Remark 4.1. The $\left(H^{1}\left(\mathbb{R}^{N}\right) \cap L^{p}\left(\mathbb{R}^{N}\right), H^{1}\left(\mathbb{R}^{N}\right) \cap L^{p}\left(\mathbb{R}^{N}\right)\right)$-uniform absorbing set obtained in Proposition 4.1 is also $\left(H^{1}\left(\mathbb{R}^{N}\right) \cap L^{p}\left(\mathbb{R}^{N}\right), L^{2}\left(\mathbb{R}^{N}\right)\right)$ - and $\left(H^{1}\left(\mathbb{R}^{N}\right) \cap\right.$ $\left.L^{p}\left(\mathbb{R}^{N}\right), L^{p}\left(\mathbb{R}^{N}\right)\right)$-uniform absorbing sets. Thus, in order to prove the existence of a uniform attractor for $\left\{U_{\sigma}(t, \tau)\right\}_{\sigma \in \mathcal{H}_{w}(g)}$, it remains to check the uniform asymptotic compactness of $\left\{U_{\sigma}(t, \tau)\right\}_{\sigma \in \mathcal{H}_{w}(g)}$.

\subsection{Existence of an $\left(H^{1}\left(\mathbb{R}^{N}\right) \cap L^{p}\left(\mathbb{R}^{N}\right), L^{2}\left(\mathbb{R}^{N}\right)\right)$-uniform attractor}

In this subsection, we assume that the external force $g$ satisfies the following hypothesis:

$\left(\mathrm{H} 2^{\prime}\right) g, \partial_{t} g \in L_{b}^{2}\left(\mathbb{R} ; L^{2}\left(\mathbb{R}^{N}\right)\right)$ and

$$
\lim _{k \rightarrow+\infty} \sup _{t \in \mathbb{R}} \int_{t}^{t+1} \int_{|x| \geq k}|g(s, x)|^{2} d x d s=0 .
$$

Lemma 4.2. For any $\tau \in \mathbb{R}$ and any bounded subset $B \subset H^{1}\left(\mathbb{R}^{N}\right) \cap L^{p}\left(\mathbb{R}^{N}\right)$, there exist $\rho_{1}>0$ and $T_{2}>\tau$ such that

$$
\left\|u_{t}(s)\right\|^{2}+2 \varepsilon\left\|\nabla u_{t}(s)\right\|^{2} \leq \rho_{1}, \forall u_{\tau} \in B, s \geq T_{2} \text {, and } \sigma \in \mathcal{H}_{w}(g) .
$$

Proof. Multiplying (1.1) by $u_{t}$ and applying the Cauchy inequality, we get

(4.8) $\left\|u_{t}\right\|^{2}+2 \varepsilon\left\|\nabla u_{t}\right\|^{2}+\frac{d}{d t}\left(\|\nabla u\|^{2}+\lambda\|u\|^{2}+2 \int_{\mathbb{R}^{N}} F(x, u) d x\right) \leq\|\sigma(t)\|^{2}$.

Integrating (4.8) from $t$ to $t+1, t \geq T_{1}$, and using (4.5) and (1.5) we have

$$
\begin{aligned}
& \int_{t}^{t+1}\left(\left\|u_{t}(s)\right\|^{2}+2 \varepsilon\left\|\nabla u_{t}(s)\right\|^{2}\right) d s \\
\leq & \|\nabla u(t)\|^{2}+\lambda\|u(t)\|^{2}+2 \int_{\mathbb{R}^{N}} F(x, u(t)) d x+\int_{t}^{t+1}\|\sigma(s)\|^{2} d s \\
\leq & \|\nabla u(t)\|^{2}+\lambda\|u(t)\|^{2}+2 \alpha_{4}\|u(t)\|_{L^{p}\left(\mathbb{R}^{N}\right)}^{p}+2\left\|\beta_{4}\right\|_{L^{1}\left(\mathbb{R}^{N}\right)}+\|\sigma\|_{b}^{2} \\
\leq & C\left(\alpha_{4}, \beta_{4},\|g\|_{b}^{2}\right) .
\end{aligned}
$$


On the other hand, differentiating (1.1) with respect to $t$, denoting $v=u_{t}$ and multiplying by $v$ in $L^{2}\left(\mathbb{R}^{N}\right)$ we get

$\frac{1}{2} \frac{d}{d t}\|v\|^{2}+\frac{\varepsilon}{2} \frac{d}{d t}\|\nabla v\|^{2}+\|\nabla v\|^{2}+\int_{\mathbb{R}^{N}} f_{u}^{\prime}(x, u) v^{2} d x+\lambda\|v\|^{2}=\int_{\mathbb{R}^{N}} \partial_{t} \sigma(t) v d x$.

Using the facts that $f_{u}^{\prime}(x, u) \geq-\ell$ and that $\int_{\mathbb{R}^{N}} \partial_{t} \sigma(t) v \leq \frac{1}{2 \lambda}\left\|\partial_{t} \sigma(t)\right\|^{2}+\frac{\lambda}{2}\|v\|^{2}$ we obtain

$$
\frac{d}{d t}\left(\|v\|^{2}+\varepsilon\|\nabla v\|^{2}\right)+2\|\nabla v\|^{2}+\lambda\|v\|^{2} \leq 2 \ell\|v\|^{2}+\frac{1}{\lambda}\left\|\partial_{t} \sigma(t)\right\|^{2},
$$

thus

$$
\frac{d}{d t}\left(\|v\|^{2}+\varepsilon\|\nabla v\|^{2}\right) \leq 2 \ell\left(\|v\|^{2}+\varepsilon\|\nabla v\|^{2}\right)+\frac{1}{\lambda}\left\|\partial_{t} \sigma(t)\right\|^{2} .
$$

From (4.9) and (4.10), using the uniform Gronwall inequality, we get the desired result.

Lemma 4.3. Let $B$ be a bounded subset in $H^{1}\left(\mathbb{R}^{N}\right) \cap L^{p}\left(\mathbb{R}^{N}\right)$. Then for any $\eta>0$, there exist $T_{\eta}>0$ and $K_{\eta}>0$ such that

$$
\int_{|x| \geq K_{\eta}}\left|U_{\sigma}(t, \tau) u_{\tau}\right|^{2} d x<\eta, \forall t \geq T_{\eta}, \forall u_{\tau} \in B, \forall \sigma \in \mathcal{H}_{w}(g) .
$$

Proof. Let $\theta: \mathbb{R}_{+} \rightarrow \mathbb{R}$ be a smooth function satisfying $\theta(s)=0,0 \leq s \leq 1$, $0 \leq \theta(s) \leq 1,1 \leq s \leq 2$, and $\theta(s)=1, s \geq 2$. It is easy to see that $\theta^{\prime}(s) \leq C$ for all $s \in[0,+\infty)$ and $\theta^{\prime}(s)=0$ for $s>2$.

Multiplying (1.1) by $\theta\left(\frac{|x|^{2}}{k^{2}}\right) u$ and integrating over $\mathbb{R}^{n}$, we obtain

$$
\begin{aligned}
& \frac{1}{2} \frac{d}{d t}\left(\int_{\mathbb{R}^{N}} \theta\left(\frac{|x|^{2}}{k^{2}}\right)|u|^{2} d x+\varepsilon \int_{\mathbb{R}^{N}} \theta\left(\frac{|x|^{2}}{k^{2}}\right)|\nabla u|^{2} d x\right) \\
& +\lambda \int_{\mathbb{R}^{N}} \theta\left(\frac{|x|^{2}}{k^{2}}\right)|u|^{2} d x+\int_{\mathbb{R}^{N}} \theta\left(\frac{|x|^{2}}{k^{2}}\right)|\nabla u|^{2} d x+\int_{\mathbb{R}^{N}} \theta\left(\frac{|x|^{2}}{k^{2}}\right) f(x, u) u d x \\
& +\int_{\mathbb{R}^{N}} \frac{2 x}{k^{2}} \theta^{\prime}\left(\frac{|x|^{2}}{k^{2}}\right) u \nabla u d x+\varepsilon \int_{\mathbb{R}^{N}} \frac{2 x}{k^{2}} \theta^{\prime}\left(\frac{|x|^{2}}{k^{2}}\right) u \nabla u_{t} d x \\
& =\int_{\mathbb{R}^{N}} \sigma(t) \theta\left(\frac{|x|^{2}}{k^{2}}\right) u d x .
\end{aligned}
$$

By (1.2), we get

$$
\begin{aligned}
\int_{\mathbb{R}^{N}} \theta\left(\frac{|x|^{2}}{k^{2}}\right) f(x, u) u & \geq \alpha_{1} \int_{\mathbb{R}^{N}} \theta\left(\frac{|x|^{2}}{k^{2}}\right)|u|^{p}-\int_{\mathbb{R}^{N}} \theta\left(\frac{|x|^{2}}{k^{2}}\right) \beta_{1}(x) \\
& \geq-\int_{|x| \geq k} \beta_{1}(x) .
\end{aligned}
$$

Because $\theta^{\prime}(s)=0$ for all $s>2$, we have

$$
\left|\int_{\mathbb{R}^{N}} \frac{2 x}{k^{2}} \theta^{\prime}\left(\frac{|x|^{2}}{k^{2}}\right) u \nabla u\right| \leq C \int_{|x| \leq \sqrt{2} k} \frac{2|x|}{k^{2}}|u||\nabla u| \leq \frac{C}{k}\left(\|u\|^{2}+\|\nabla u\|^{2}\right),
$$


and similarly,

$$
\left|\varepsilon \int_{\mathbb{R}^{N}} \frac{2 x}{k^{2}} \theta^{\prime}\left(\frac{|x|^{2}}{k^{2}}\right) u \nabla u_{t}\right| \leq \frac{C}{k}\left(\|u\|^{2}+\varepsilon^{2}\left\|\nabla u_{t}\right\|^{2}\right) .
$$

By the Cauchy inequality, we see that

$$
\begin{aligned}
\left|\int_{\mathbb{R}^{N}} \sigma(t) \theta\left(\frac{|x|^{2}}{k^{2}}\right) u\right| & \leq \frac{\lambda}{2} \int_{\mathbb{R}^{N}} \theta\left(\frac{|x|^{2}}{k^{2}}\right)|u|^{2}+\frac{1}{2 \lambda} \int_{\mathbb{R}^{N}} \theta\left(\frac{|x|^{2}}{k^{2}}\right)|\sigma(t)|^{2} \\
& \leq \frac{\lambda}{2} \int_{\mathbb{R}^{N}} \theta\left(\frac{|x|^{2}}{k^{2}}\right)|u|^{2}+\frac{1}{2 \lambda} \int_{|x| \geq k}|\sigma(t)|^{2} .
\end{aligned}
$$

Combining (4.11)-(4.15), we deduce that

$$
\begin{aligned}
& \frac{d}{d t}\left(\int_{\mathbb{R}^{N}} \theta\left(\frac{|x|^{2}}{k^{2}}\right)|u|^{2}+\varepsilon \int_{\mathbb{R}^{N}} \theta\left(\frac{|x|^{2}}{k^{2}}\right)|\nabla u|^{2}\right) \\
& +\delta\left(\int_{\mathbb{R}^{N}} \theta\left(\frac{|x|^{2}}{k^{2}}\right)|u|^{2}+\varepsilon \int_{\mathbb{R}^{N}} \theta\left(\frac{|x|^{2}}{k^{2}}\right)|\nabla u|^{2}\right) \\
\leq & \frac{1}{\lambda} \int_{|x| \geq k}|\sigma(t)|^{2}+2 \int_{|x| \geq k} \beta_{1}(x)+\frac{C}{k}\left(\|u\|^{2}+\|\nabla u\|^{2}+\varepsilon^{2}\left\|\nabla u_{t}\right\|^{2}\right) .
\end{aligned}
$$

Multiplying (4.16) by $e^{\delta t}$ and integrating from $T^{*}$ to $t$, where $T^{*}=\max \left\{T_{1}, T_{2}\right\}$, we find that

$$
\begin{aligned}
& \int_{\mathbb{R}^{N}} \theta\left(\frac{|x|^{2}}{k^{2}}\right)|u(t)|^{2}+\varepsilon \int_{\mathbb{R}^{N}} \theta\left(\frac{|x|^{2}}{k^{2}}\right)|\nabla u(t)|^{2} \\
\leq & e^{-\delta\left(t-T^{*}\right)}\left(\left\|u\left(T^{*}\right)\right\|^{2}+\varepsilon\left\|\nabla u\left(T^{*}\right)\right\|^{2}\right)+\frac{e^{-\delta t}}{\lambda} \int_{T^{*}}^{t} \int_{|x| \geq k} e^{\delta s}|\sigma(s)|^{2} \\
& +2 e^{-\delta t} \int_{T^{*}}^{t} \int_{|x| \geq k} e^{\delta s} \beta_{1}(x)+\frac{C e^{-\delta t}}{k} \int_{T^{*}}^{t}\left(e^{\delta s}\left(\|u\|^{2}+\|\nabla u\|^{2}+\varepsilon^{2}\left\|\nabla u_{t}\right\|^{2}\right)\right) .
\end{aligned}
$$

Since $\beta_{1} \in L^{1}\left(\mathbb{R}^{N}\right)$, we have

$$
\limsup _{t \rightarrow+\infty} \limsup _{k \rightarrow+\infty} e^{-\delta t} \int_{\tau}^{t} \int_{|x| \geq k} e^{\delta s} \beta_{1}(x)=0 .
$$

Using the arguments in (3.19) and assumption (4.6) we get

$$
\limsup _{t \rightarrow+\infty} \limsup _{k \rightarrow+\infty} e^{-\delta t} \int_{\tau}^{t} \int_{|x| \geq k} e^{\delta s}|\sigma(s)|^{2}=0 .
$$

By (4.5) and (4.7), we have

$$
e^{-\delta\left(t-T^{*}\right)}\left(\left\|u\left(T^{*}\right)\right\|^{2}+\varepsilon\left\|\nabla u\left(T^{*}\right)\right\|^{2}\right) \leq e^{-\delta\left(t-T^{*}\right)} \rho_{0} \rightarrow 0 \text { as } t \rightarrow+\infty,
$$


and

$$
\begin{aligned}
& \frac{C e^{-\delta t}}{k} \int_{T^{*}}^{t}\left(e^{\delta s}\left(\|u\|^{2}+\|\nabla u\|^{2}+\varepsilon^{2}\left\|\nabla u_{t}\right\|^{2}\right)\right) \\
\leq & \frac{C e^{-\delta t}}{k} \int_{T^{*}}^{t}\left(e^{\delta s}\left(\rho_{0}+\rho_{1}\right)\right) \\
\leq & \frac{C\left(\rho_{0}, \rho_{1}\right)}{k} \rightarrow 0 \text { as } k \rightarrow+\infty .
\end{aligned}
$$

From (4.17)-(4.21), we can take $T_{\eta}$ and $K_{\eta}>0$ large enough such that

$$
\int_{|x| \geq K_{\eta}}\left(|u(t)|^{2}+\varepsilon|\nabla u(t)|^{2}\right)<\eta \text { for all } t \geq T_{\eta}, u_{\tau} \in B \text { and } \sigma \in \mathcal{H}_{w}(g) .
$$

This completes the proof.

Theorem 4.4. Assume that $f$ satisfies (H1) and $g$ satisfies (H2'). Then the family of processes $\left\{U_{\sigma}(t, \tau)\right\}_{\sigma \in \mathcal{H}_{w}(g)}$ possesses an $\left(H^{1}\left(\mathbb{R}^{N}\right) \cap L^{p}\left(\mathbb{R}^{N}\right)\right.$, $L^{2}\left(\mathbb{R}^{N}\right)$ )-uniform attractor $\mathcal{A}_{2}$. Moreover, we have

$$
\mathcal{A}_{2}=\bigcup_{\sigma \in \mathcal{H}_{w}(g)} \mathcal{K}_{\sigma}(s), \forall s \in \mathbb{R},
$$

where $\mathcal{K}_{\sigma}(s)$ is the kernel section at time $s$ of the process $U_{\sigma}(t, \tau)$.

Proof. By Proposition 4.1, $\left\{U_{\sigma}(t, \tau)\right\}$ has an $\left(H^{1}\left(\mathbb{R}^{N}\right) \cap L^{p}\left(\mathbb{R}^{N}\right), L^{2}\left(\mathbb{R}^{N}\right)\right)$ uniform absorbing set. It remains to show that $\left\{U_{\sigma}(t, \tau)\right\}$ is $\left(H^{1}\left(\mathbb{R}^{N}\right) \cap\right.$ $L^{p}\left(\mathbb{R}^{N}\right), L^{2}\left(\mathbb{R}^{N}\right)$ )-uniformly asymptotically compact. Fix $\tau \in \mathbb{R}$, let $\left\{x_{n}\right\} \subset$ $H^{1}\left(\mathbb{R}^{N}\right) \cap L^{p}\left(\mathbb{R}^{N}\right)$ be a bounded sequence, $\left\{t_{n}\right\}$ be a sequence such that $\lim _{n \rightarrow \infty} t_{n}=\infty$ and $\left\{\sigma_{n}\right\} \subset \mathcal{H}_{w}(g)$, we have to show that $\left\{U_{\sigma_{n}}\left(t_{n}, \tau\right) x_{n}\right\}$ is precompact in $L^{2}\left(\mathbb{R}^{N}\right)$. We will prove that, for any $\eta>0$, there exists a finite covering balls with radii $\eta$ for $\left\{U_{\sigma_{n}}\left(t_{n}, \tau\right) x_{n}\right\}$. Since $t_{n} \rightarrow+\infty$, we can choose $N$ large enough such that $t_{n} \geq T_{\eta}$ for all $n \geq N$, where $T_{\eta}$ is stated in Lemma 4.3. From Lemma 4.3, there exists $K_{\eta}>0$ such that

$$
\left\|U_{\sigma_{n}}\left(t_{n}, \tau\right) x_{n}\right\|_{L^{2}\left(B_{K_{\eta}}^{c}\right)}^{2}<\frac{\eta}{4}, \forall n \geq N,
$$

where $B_{K_{\eta}}^{c}=\left\{x \in \mathbb{R}^{N}:|x|>K_{\eta}\right\}$. On the other hand, from Proposition 4.1, the sequence $\left\{U_{\sigma_{n}}\left(t_{n}, \tau\right) x_{n}\right\}$ is bounded in $H^{1}\left(B_{K_{\eta}}\right)$; taking into account that $H^{1}\left(B_{K_{\eta}}\right) \hookrightarrow L^{2}\left(B_{K_{\eta}}\right)$ compactly, we see that

$$
\begin{aligned}
& \left\{U_{\sigma_{n}}\left(t_{n}, \tau\right) x_{n}\right\} \text { has a finite covering by balls with radii less than } \\
& \quad \frac{\eta}{4} \text { in } L^{2}\left(B_{K_{\eta}}\right) .
\end{aligned}
$$

Combining (4.22) and (4.23) we obtain the existence of a finite covering by balls with radii $\eta$ for $\left\{U_{\sigma_{n}}\left(t_{n}, \tau\right) x_{n}\right\}$. Hence we get the existence of an $\left(H^{1}\left(\mathbb{R}^{N}\right) \cap\right.$ $L^{p}\left(\mathbb{R}^{N}\right), L^{2}\left(\mathbb{R}^{N}\right)$ )-uniform attractor $\mathcal{A}_{2}$. The structure of $\mathcal{A}_{2}$ follows directly from Theorem 2.1. 


\subsection{Existence of an $\left(H^{1}\left(\mathbb{R}^{N}\right) \cap L^{p}\left(\mathbb{R}^{N}\right), L^{p}\left(\mathbb{R}^{N}\right)\right)$-uniform attractor}

To prove the existence of $\left(H^{1}\left(\mathbb{R}^{N}\right) \cap L^{p}\left(\mathbb{R}^{N}\right), L^{p}\left(\mathbb{R}^{N}\right)\right)$ and $\left(H^{1}\left(\mathbb{R}^{N}\right) \cap\right.$ $L^{p}\left(\mathbb{R}^{N}\right), H^{1}\left(\mathbb{R}^{N}\right) \cap L^{p}\left(\mathbb{R}^{N}\right)$ )-uniform attractors, we assume that the external force $g$ satisfies the following hypothesis:

$(\mathrm{H} 2 ") g \in L^{\infty}\left(\mathbb{R} ; L^{2}\left(\mathbb{R}^{N}\right)\right), \partial_{t} g \in L_{b}^{2}\left(\mathbb{R} ; L^{2}\left(\mathbb{R}^{N}\right)\right)$ and

$$
\lim _{k \rightarrow+\infty} \sup _{t \in \mathbb{R}} \int_{t}^{t+1} \int_{|x| \geq k}|g(s, x)|^{2} d x d s=0 .
$$

It is obvious that if $g \in L^{\infty}\left(\mathbb{R} ; L^{2}\left(\mathbb{R}^{N}\right)\right)$, then $g \in L_{b}^{2}\left(\mathbb{R} ; L^{2}\left(\mathbb{R}^{N}\right)\right)$. Thus, all estimates in previous sections are still valid here. It is noticed that

$$
\sigma \in L^{\infty}\left(\mathbb{R} ; L^{2}\left(\mathbb{R}^{N}\right)\right) \text { for all } \sigma \in \mathcal{H}_{w}(g) .
$$

From now on, for the shake of brevity, we will use the notations

$$
\Omega(\Phi)=\left\{x \in \mathbb{R}^{N}: \Phi \text { is true }\right\} \text {, where } \Phi \text { is a logical condition, }
$$

and

$$
\|\sigma\|_{\infty}=\|\sigma\|_{L^{\infty}\left(\mathbb{R} ; L^{2}\left(\mathbb{R}^{N}\right)\right)} \text { for all } \sigma \in \mathcal{H}_{w}(g) .
$$

Lemma 4.5. For any $\tau \in \mathbb{R}$, any bounded subset $B \subset H^{1}\left(\mathbb{R}^{N}\right) \cap L^{p}\left(\mathbb{R}^{N}\right)$ and any $\eta>0$, there exist $T$ and $M_{0}$ such that

$\operatorname{mes}\left(\Omega\left(\left|U_{\sigma}(t, \tau) u_{\tau}\right| \geq M\right)\right) \leq \eta$ for all $t>T, M>M_{0}, u_{\tau} \in B$ and $\sigma \in \mathcal{H}_{w}(g)$, where mes $(G)$ is the Lebesgue measure of a subset $G$ of $\mathbb{R}^{N}$.

Proof. From (4.5) we see that for any $t \geq T_{1}$, any $u_{\tau} \in B$ and any $\sigma \in \mathcal{H}_{w}(g)$, we have

$$
\begin{aligned}
\rho_{0} & \geq \int_{\mathbb{R}^{N}}\left|U_{\sigma}(t, \tau) u_{\tau}\right|^{2} \\
& \geq \int_{\Omega\left(\left|U_{\sigma}(t, \tau) u_{\tau}\right| \geq M\right)}\left|U_{\sigma}(t, \tau) u_{\tau}\right|^{2} \\
& \geq M^{2} \operatorname{mes}\left(\Omega\left(\left|U_{\sigma}(t, \tau) u_{\tau}\right| \geq M\right)\right) .
\end{aligned}
$$

Thus, we arrive at (4.25) by choosing $T=T_{1}$ and $M_{0}=\left(\frac{\rho_{0}}{\eta}\right)^{1 / 2}$.

Lemma 4.6. For any $\tau \in \mathbb{R}$, any bounded subset $B \subset H^{1}\left(\mathbb{R}^{N}\right) \cap L^{p}\left(\mathbb{R}^{N}\right)$ and any $\eta>0$, there exist $T>\tau$ and $M_{0}>0$ such that

$\int_{\Omega(|u| \geq M)}\left((|u|-M)^{2}+\varepsilon|\nabla u|^{2}\right) \leq \eta$ for all $t>T, u_{\tau} \in B, M \geq M_{0}$ and $\sigma \in \mathcal{H}_{w}(g)$.

Proof. Denote

$$
(u-M)_{+}= \begin{cases}u-M & \text { if } u \geq M \\ 0 & \text { if } u \leq M\end{cases}
$$


and $\Omega_{M}=\Omega(u \geq M)$. Multiplying (1.1) by $(u-M)_{+}$and integrating over $\mathbb{R}^{N}$, we get

$$
\begin{aligned}
& \frac{1}{2} \frac{d}{d t} \int_{\Omega_{M}}\left(|u-M|^{2}+\varepsilon|\nabla u|^{2}\right)+\int_{\Omega_{M}}|\nabla u|^{2}+\int_{\Omega_{M}} f(x, u)(u-M) \\
& +\lambda \int_{\Omega_{M}} u(u-M) \\
= & \int_{\Omega_{M}} \sigma(t)(u-M) .
\end{aligned}
$$

Using assumptions (1.2)-(1.3), the fact that $u \geq M$ in $\Omega_{M}$, and Young's inequality, we have

$$
\begin{aligned}
& \int_{\Omega_{M}} f(x, u)(u-M) \\
= & \int_{\Omega_{M}} f(x, u) u d x-\int_{\Omega_{M}} f(x, u) M \\
\geq & \int_{\Omega_{M}}\left[\alpha_{1} u^{p}-\beta_{1}(x)\right]-M \int_{\Omega_{M}}\left[\alpha_{2} u^{p-1}+\beta_{2}(x)\right] \\
\geq & \int_{\Omega_{M}}\left[\alpha_{1} u^{p}-\beta_{1}(x)\right]-M \int_{\Omega_{M}} \alpha_{2} u^{p-1}-\int_{\Omega_{M}} \beta_{2}(x) u \\
\geq & \frac{\alpha_{1}}{2} \int_{\Omega_{M}} u^{p}-\int_{\Omega_{M}} \beta_{1}(x)-C_{1} \int_{\Omega_{M}} \beta_{2}(x)^{p^{\prime}}-C_{2} \operatorname{mes}\left(\Omega_{M}\right) .
\end{aligned}
$$

By the Cauchy inequality and hypothesis (4.24), we get

$$
\left.\int_{\Omega_{M}} \sigma(t)(u-M) \leq \frac{\|\sigma\|_{\infty}^{2}}{2 \lambda} \operatorname{mes}\left(\Omega_{M}\right)\right)+\frac{\lambda}{2} \int_{\Omega_{M}}|u-M|^{2} .
$$

Combining (4.26)-(4.28) and the fact that $\int_{\Omega_{M}} u(u-M) \geq \int_{\Omega_{M}}|u-M|^{2}$, in particular, we obtain

$$
\begin{aligned}
& \frac{d}{d t} \int_{\Omega_{M}}\left(|u-M|^{2}+\varepsilon|\nabla u|^{2}\right)+\delta \int_{\Omega_{M}}\left(|u-M|^{2}+\varepsilon|\nabla u|^{2}\right) \\
\leq & C\left(\operatorname{mes}\left(\Omega_{M}\right)+\int_{\Omega_{M}} \beta_{1}(x)+\int_{\Omega_{M}} \beta_{2}(x)^{p^{\prime}}\right) .
\end{aligned}
$$

Thus, by the Gronwall inequality we find that

$$
\begin{aligned}
& \int_{\Omega_{M}}\left(|u(t)-M|^{2}+\varepsilon|\nabla u(t)|^{2}\right) \\
\leq & e^{-\delta(t-\tau)}\left(\left\|u_{\tau}-M\right\|^{2}+\varepsilon\left\|\nabla u_{\tau}\right\|^{2}\right)+C\left(\operatorname{mes}\left(\Omega_{M}\right)+\int_{\Omega_{M}} \beta_{1}(x)\right. \\
& \left.+\int_{\Omega_{M}} \beta_{2}(x)^{p^{\prime}}\right) .
\end{aligned}
$$


Using Lemma 4.5 and the facts that $\beta_{1} \in L^{1}\left(\mathbb{R}^{N}\right), \beta_{2} \in L^{p^{\prime}}\left(\mathbb{R}^{N}\right)$, and $u_{\tau}$ belongs to the bounded set $B$, we obtain

$$
\int_{\Omega_{M}}\left(|u-M|^{2}+\varepsilon|\nabla u|^{2}\right) \leq \eta \text { when } t \text { and } M \text { large enough. }
$$

Repeating the above arguments and replacing $(u-M)_{+}$by $(u+M)_{-}$, where

$$
(u+M)_{-}= \begin{cases}u+M & \text { if } u \leq-M \\ 0 & \text { if } u \geq-M\end{cases}
$$

we get

$$
\int_{\Omega(u \leq-M)}\left((u+M)^{2}+\varepsilon|\nabla u|^{2}\right) \leq \eta
$$

for $t$ and $M$ large enough. Combining (4.29) and (4.30) completes the proof.

Lemma $4.7([9])$. Let $p \geq 2$ and $\left\{U_{\sigma}(t, \tau)\right\}_{\sigma \in \mathcal{H}_{w}(g)}$ be a family of processes satisfying the following assumptions:

(i) $\left\{U_{\sigma(t, \tau)}\right\}_{\sigma \in \mathcal{H}_{w}(g)}$ has an $\left(H^{1}\left(\mathbb{R}^{N}\right) \cap L^{p}\left(\mathbb{R}^{N}\right), L^{2}\left(\mathbb{R}^{N}\right)\right)$-uniform attractor;

(ii) $\left\{U_{\sigma}(t, \tau)\right\}_{\sigma \in \mathcal{H}_{w}(g)}$ has an $\left(H^{1}\left(\mathbb{R}^{N}\right) \cap L^{p}\left(\mathbb{R}^{N}\right), L^{p}\left(\mathbb{R}^{N}\right)\right)$-uniform absorbing set;

(iii) for any $\eta>0$ and any bounded set $B \subset H^{1}\left(\mathbb{R}^{N}\right) \cap L^{p}\left(\mathbb{R}^{N}\right)$, there exist $M$ and $T$ such that

$$
\int_{\Omega\left(\left|U_{\sigma}(t, \tau) u_{\tau}\right| \geq M\right)}\left|U_{\sigma}(t, \tau) u_{\tau}\right|^{p} \leq C \eta \quad \text { for all } u_{\tau} \in B, t \geq T \text { and } \sigma \in \mathcal{H}_{w}(g),
$$

where $C$ is independent of $\eta, u_{\tau}, t$ and $\sigma$. Then $\left\{U_{\sigma}(t, \tau)\right\}_{\sigma \in \mathcal{H}_{w}(g)}$ has an $\left(H^{1}\left(\mathbb{R}^{N}\right) \cap L^{p}\left(\mathbb{R}^{N}\right), L^{p}\left(\mathbb{R}^{N}\right)\right)$-uniform attractor.

Theorem 4.8. Assume that $f$ satisfies (H1) and $g$ satisfies (H2"). Then the family of processes $\left\{U_{\sigma}(t, \tau)\right\}_{\sigma \in \mathcal{H}_{w}(g)}$ associated to problem (1.1) possesses an $\left(H^{1}\left(\mathbb{R}^{N}\right) \cap L^{p}\left(\mathbb{R}^{N}\right), L^{p}\left(\mathbb{R}^{N}\right)\right)$-uniform attractor $\mathcal{A}_{p}$, and

$$
\mathcal{A}_{p}=\bigcup_{\sigma \in \mathcal{H}_{w}(g)} \mathcal{K}_{\sigma}(s) \text { for all } s \in \mathbb{R} .
$$

Proof. By Proposition 4.1, Theorem 4.4 and Lemma 4.7, we only need to show that for any $\eta>0$ and any bounded subset $B \subset H^{1}\left(\mathbb{R}^{N}\right) \cap L^{p}\left(\mathbb{R}^{N}\right)$, there exist $M>0$ and $T \geq \tau$ such that

$$
\int_{\Omega(|u(t)| \geq M)}|u(t)|^{p} \leq C \eta
$$

for all $t \geq T, u_{\tau} \in B$ and $\sigma \in \mathcal{H}_{w}(g)$. Indeed, for any $\sigma \in \mathcal{H}_{w}(g)$, by taking $(u-M)_{+}$as a test function in (1.1), we have

$$
\int_{\Omega_{M}} u_{t}(u-M)+\varepsilon \int_{\Omega_{M}} \nabla u_{t} \nabla u+\int_{\Omega_{M}}|\nabla u|^{2}+\int_{\Omega_{M}} f(x, u)(u-M)
$$


$=\int_{\Omega_{M}} \sigma(t)(u-M)$

Using estimate (4.27) we get

$$
\begin{aligned}
& \frac{\alpha_{1}}{2} \int_{\Omega(u \geq M)}|u(t)|^{p} \\
\leq & \int_{\Omega_{M}}\left|u_{t}\right||u-M|+\varepsilon \int_{\Omega_{M}}\left|\nabla u_{t}\right||\nabla u|+\int_{\Omega_{M}}|\sigma(t)||u-M| \\
& +\int_{\Omega_{M}} \beta_{1}(x)+C_{1} \int_{\Omega_{M}} \beta_{2}(x)^{p^{\prime}}+C_{2} \operatorname{mes}\left(\Omega_{M}\right) \\
\leq & \left\|u_{t}(t)\right\|\left(\int_{\Omega_{M}}|u-M|^{2}\right)^{1 / 2}+\varepsilon\left\|\nabla u_{t}(t)\right\|\left(\int_{\Omega_{M}}|\nabla u|^{2}\right)^{1 / 2} \\
& +\|\sigma\|_{\infty}\left(\int_{\Omega_{M}}|u-M|^{2}\right)^{1 / 2}+\int_{\Omega_{M}} \beta_{1}(x)+C_{1} \int_{\Omega_{M}} \beta_{2}(x)^{p^{\prime}}+C_{2} \operatorname{mes}\left(\Omega_{M}\right) .
\end{aligned}
$$

Using Lemma 4.2 we deduce that

$$
\begin{aligned}
\int_{\Omega(u \geq M)}|u(t)|^{p} \leq & C\left(\left(\int_{\Omega_{M}}|u-M|^{2}\right)^{1 / 2}+\left(\int_{\Omega_{M}}|\nabla u|^{2}\right)^{1 / 2}\right. \\
& \left.+\int_{\Omega_{M}} \beta_{1}(x)+\int_{\Omega_{M}} \beta_{2}(x)^{p^{\prime}}+\operatorname{mes}\left(\Omega_{M}\right)\right)
\end{aligned}
$$

when $t$ is large enough. Since $\beta_{1} \in L^{1}\left(\mathbb{R}^{N}\right)$ and $\beta_{2} \in L^{p^{\prime}}\left(\mathbb{R}^{N}\right)$, we have

$$
\int_{\Omega_{M}} \beta_{1}(x)+\int_{\Omega_{M}} \beta_{2}(x)^{p^{\prime}}<\eta
$$

if $M$ is large enough. Taking into account Lemmas 4.5 and 4.6 , there exist $T_{0}$ and $M_{0}$ such that

$$
\int_{\Omega(u(t) \geq M)}|u(t)|^{p} \leq C \eta
$$

for all $t \geq T_{0}, u_{\tau} \in B, \sigma \in \mathcal{H}_{w}(g)$ and $M \geq M_{0}$. Similarly, replacing $(u-M)_{+}$ by $(u+M)_{-}$, we can deduce that

$$
\int_{\Omega(u(t) \leq-M)}|u(t)|^{p} \leq C \eta .
$$

Hence we obtain (4.31) and this completes the proof.

\subsection{Existence of an $\left(H^{1}\left(\mathbb{R}^{N}\right) \cap L^{p}\left(\mathbb{R}^{N}\right), H^{1}\left(\mathbb{R}^{N}\right) \cap L^{p}\left(\mathbb{R}^{N}\right)\right)$-uniform attractor}

We first prove the following lemma, which is very useful in verifying the uniform asymptotic compactness of the family of processes $\left\{U_{\sigma}(t, \tau)\right\}_{\sigma \in \mathcal{H}_{w}(g)}$. 
Lemma 4.9. If a family of processes $\left\{U_{\sigma}(t, \tau)\right\}_{\sigma \in \Sigma}$ satisfies the uniform ( $X$, $Y)$-condition $(\mathrm{C})$, that is, for any fixed $\tau \in \mathbb{R}, B \in \mathcal{B}(X)$, and any $\eta>0$, there exist $T \geq \tau$ and a finite dimensional subspace $Y_{1}$ of $Y$ such that:

(i) $P\left(\bigcup_{\sigma \in \Sigma} \bigcup_{t \geq T} U_{\sigma}(t, \tau) B\right)$ is bounded in $Y_{1}$,

(ii) $\left\|\left(I d_{Y}-P\right) y\right\|_{Y} \leq \eta, \forall y \in \bigcup_{\sigma \in \Sigma} \bigcup_{t>T} U_{\sigma}(t, \tau) B$,

where $P: Y \rightarrow Y_{1}$ is a bounded projector, $I d_{Y}$ is the identity, then $\left\{U_{\sigma}(t, \tau)\right\}_{\sigma \in \Sigma}$ is $(X, Y)$-uniformly (w.r.t $\sigma \in \Sigma$ ) asymptotically compact.

Proof. Let $\left\{t_{n}\right\} \subset[\tau,+\infty), t_{n} \rightarrow+\infty,\left\{\sigma_{n}\right\} \subset \Sigma$ and $\left\{x_{n}\right\} \subset B$. We have to prove that $\left\{U_{\sigma_{n}}\left(t_{n}, \tau\right) x_{n}\right\}$ is precompact in $Y$. Take $N$ such that $t_{n} \geq T$ for all $n \geq N$. Let $\eta>0$ be arbitrary. By (i), we see that $P\left(\left\{U_{\sigma_{n}}\left(t_{n}, \tau\right) x_{n}\right\}_{n \geq N}\right)$ is bounded in $Y_{1}$. Since $Y_{1}$ is finite dimensional, without loss of generality, we can assume that $\left\{P U_{\sigma_{n}}\left(t_{n}, \tau\right) x_{n}\right\}_{n \geq N}$ is a Cauchy sequence in $Y$. Thus, there exists $N_{1}>N$ such that

$$
\left\|P U_{\sigma_{n}}\left(t_{n}, \tau\right) x_{n}-P U_{\sigma_{m}}\left(t_{m}, \tau\right) x_{m}\right\|_{Y} \leq \frac{\eta}{3} \text { for all } m, n \geq N_{1} .
$$

On the other hand, by (ii), we conclude that there exists $N_{2}>N$ such that

$$
\left\|\left(I d_{Y}-P\right) U_{\sigma_{n}}\left(t_{n}, \tau\right) x_{n}\right\|_{Y} \leq \frac{\eta}{3} \text { for all } n \geq N_{2} .
$$

Now, taking $N_{*}=\max \left\{N_{1}, N_{2}\right\}$, we have

$$
\begin{aligned}
& \left\|U_{\sigma_{n}}\left(t_{n}, \tau\right) x_{n}-U_{\sigma_{m}}\left(t_{m}, \tau\right) x_{m}\right\|_{Y} \\
\leq & \left\|P U_{\sigma_{n}}\left(t_{n}, \tau\right) x_{n}-P U_{\sigma_{m}}\left(t_{m}, \tau\right) x_{m}\right\|_{Y}+\left\|\left(I d_{Y}-P\right) U_{\sigma_{n}}\left(t_{n}, \tau\right) x_{n}\right\|_{Y} \\
& +\left\|\left(I d_{Y}-P\right) U_{\sigma_{m}}\left(t_{m}, \tau\right) x_{m}\right\|_{Y} \\
\leq & \eta \text { for all } n, m \geq N_{*} .
\end{aligned}
$$

This means that $\left\{U_{\sigma_{n}}\left(t_{n}, \tau\right) x_{n}\right\}$ is a Cauchy sequence in $Y$. The proof is complete.

Lemma 4.10. Assume that $2 \leq q<\infty$ and $\left\{U_{\sigma}(t, \tau)\right\}_{\sigma \in \Sigma}$ has an $\left(H^{1}\left(\mathbb{R}^{N}\right) \cap\right.$ $\left.L^{p}\left(\mathbb{R}^{N}\right), L^{q}\left(\mathbb{R}^{N}\right)\right)$-uniform attractor. Then, for any $\eta>0$, any $\tau \in \mathbb{R}$ and any bounded subset $B \subset H^{1}\left(\mathbb{R}^{N}\right) \cap L^{p}\left(\mathbb{R}^{N}\right)$, there exist $T \geq \tau$ and $m_{0} \in \mathbb{N}$ such that

$$
\int_{\mathbb{R}^{N}}\left|\left(I-P_{m}\right) U_{\sigma}(t, \tau) u_{\tau}\right|^{q} \leq C \eta \text { for any } t \geq T, u_{\tau} \in B, m \geq m_{0}, \sigma \in \Sigma,
$$

where $P_{m}$ is the canonical projection of $L^{q}\left(\mathbb{R}^{N}\right)$ onto an m-dimensional subspace.

Proof. Let $\mathcal{A}$ be the $\left(H^{1}\left(\mathbb{R}^{N}\right) \cap L^{p}\left(\mathbb{R}^{N}\right), L^{q}\left(\mathbb{R}^{N}\right)\right)$-uniform attractor of

$$
\left\{U_{\sigma}(t, \tau)\right\}_{\sigma \in \Sigma} \text {. }
$$


Then for any $\eta>0$, any $\tau \in \mathbb{R}$ and any bounded subset $B \subset H^{1}\left(\mathbb{R}^{N}\right) \cap L^{p}\left(\mathbb{R}^{N}\right)$, there exists $T_{0}$ such that

$$
\bigcup_{t \geq T_{0}} \bigcup_{\sigma \in \Sigma} U_{\sigma}(t, \tau) B \subset \mathcal{N}_{L^{q}}(\mathcal{A}, \eta)
$$

where $\mathcal{N}_{L^{q}}(\mathcal{A}, \eta)$ is the $\eta$-neighborhood of $\mathcal{A}$ in $L^{q}\left(\mathbb{R}^{N}\right)$. Since $\mathcal{A}$ is compact in $L^{q}\left(\mathbb{R}^{N}\right)$, there exist $n \in \mathbb{N}$ and $v_{i} \in L^{q}\left(\mathbb{R}^{N}\right), i=1, \ldots, n$, such that

$$
\bigcup_{t \geq T_{0}} \bigcup_{\sigma \in \Sigma} U_{\sigma}(t, \tau) B \subset \bigcup_{i=1}^{n} \mathcal{N}_{L^{q}}\left(v_{i}, \eta\right)
$$

For each $v_{i}$ there is an $m_{i}$ such that

$$
\int_{\mathbb{R}^{N}}\left|\left(I-P_{m}\right) v_{i}\right|^{q} \leq \eta \text { for all } m \geq m_{i}
$$

Taking $m_{0}=\max \left\{m_{1}, \ldots, m_{n}\right\}$. Denote $Q_{m_{0}}=I-P_{m_{0}}$ for any $t \geq T_{0}$, any $u_{\tau} \in B$, and any $\sigma \in \mathcal{H}_{w}(g)$ there exists some $v_{i}$ such that

$$
\begin{aligned}
\int_{\mathbb{R}^{N}}\left|Q_{m_{0}} U_{\sigma}(t, \tau) u_{\tau}\right|^{q} & =\int_{\mathbb{R}^{N}}\left|Q_{m_{0}} U_{\sigma}(t, \tau) u_{\tau}-Q_{m_{0}} v_{i}+Q_{m_{0}} v_{i}\right|^{q} \\
& \leq 2^{q} \int_{\mathbb{R}^{N}}\left|Q_{m_{0}} U_{\sigma}(t, \tau) u_{\tau}-Q_{m_{0}} v_{i}\right|^{q}+2^{q} \int_{\mathbb{R}^{N}}\left|Q_{m_{0}} v_{i}\right|^{q} \\
& \leq 2^{q} C_{q} \int_{\mathbb{R}^{N}}\left|U_{\sigma}(t, \tau) u_{\tau}-v_{i}\right|^{q}+2^{q} \int_{\mathbb{R}^{N}}\left|Q_{m_{0}} v_{i}\right|^{q} \\
& \leq 2^{q}\left(C_{q}+1\right) \eta
\end{aligned}
$$

where $C_{q}$ depends only on $q$. This completes the proof.

We are now ready to prove the main result of this section.

Theorem 4.11. Assume that conditions (H1) and (H2") hold. Then the family of processes $\left\{U_{\sigma}(t, \tau)\right\}_{\sigma \in \mathcal{H}_{w}(g)}$ generated by problem (1.1) possesses an $\left(H^{1}\left(\mathbb{R}^{N}\right) \cap L^{p}\left(\mathbb{R}^{N}\right), H^{1}\left(\mathbb{R}^{N}\right) \cap L^{p}\left(\mathbb{R}^{N}\right)\right)$-uniform attractor $\mathcal{A}_{\mathcal{H}_{w}(g)}$. Moreover,

$$
\mathcal{A}_{\mathcal{H}_{w}(g)}=\bigcup_{\sigma \in \mathcal{H}_{w}(g)} \mathcal{K}_{\sigma}(s), \forall s \in \mathbb{R}
$$

Proof. Since $H^{1}\left(\mathbb{R}^{N}\right)$ is separable, we can choose a set $\left\{w_{1}, w_{2}, \ldots\right\}$ which forms an orthogonal basis in both $L^{2}\left(\mathbb{R}^{N}\right)$ and $H^{1}\left(\mathbb{R}^{N}\right)$. Let $H_{m}=\operatorname{span}\left\{w_{1}, w_{2}, \ldots\right.$, $\left.w_{m}\right\}, P_{m}$ be the canonical projector on $H_{m}$ and $I$ be the identity. Then for any $u \in H^{1}\left(\mathbb{R}^{N}\right), u$ has a unique decomposition: $u=u_{1}+u_{2}$, where $u_{1}=P_{m} u \in$ $H_{m}$ and $u_{2}=\left(I-P_{m}\right) u$. Let $\eta>0$ be arbitrary. Taking $u_{2}$ as a test function in (1.1), we obtain

$$
\frac{1}{2} \frac{d}{d t}\left(\left\|u_{2}\right\|^{2}+\varepsilon\left\|\nabla u_{2}\right\|^{2}\right)+\left\|\nabla u_{2}\right\|^{2}+\int_{\mathbb{R}^{N}} f(x, u) u_{2}+\lambda\left\|u_{2}\right\|^{2}=\int_{\mathbb{R}^{N}} \sigma(t) u_{2},
$$


thus,

$$
\begin{aligned}
& \frac{d}{d t}\left(\left\|u_{2}\right\|^{2}+\varepsilon\left\|\nabla u_{2}\right\|^{2}\right)+2\left\|\nabla u_{2}\right\|^{2}+2 \lambda\left\|u_{2}\right\|^{2} \\
\leq & 2\|f(x, u)\|_{L^{p^{\prime}\left(\mathbb{R}^{N}\right)}}\left\|u_{2}\right\|_{L^{p}\left(\mathbb{R}^{N}\right)}+2\|\sigma(t)\|\left\|u_{2}\right\| .
\end{aligned}
$$

Using (1.3), we get $|f(x, u)|^{p^{\prime}} \leq C\left(|u|^{p}+\beta_{2}(x)^{p^{\prime}}\right)$, hence $\|f(x, u)\|_{L^{p^{\prime}}\left(\mathbb{R}^{N}\right)}$ is bounded when $t$ large enough due to Proposition 4.1. Since $\left\{U_{\sigma}(t, \tau)\right\}_{\sigma \in \Sigma}$ has $\left(H^{1}\left(\mathbb{R}^{N}\right) \cap L^{p}\left(\mathbb{R}^{N}\right), L^{2}\left(\mathbb{R}^{N}\right)\right)$ and $\left(H^{1}\left(\mathbb{R}^{N}\right) \cap L^{p}\left(\mathbb{R}^{N}\right), L^{p}\left(\mathbb{R}^{N}\right)\right)$-uniform attractors, by Lemma 4.10 we get $m^{*}$ such that

$$
\left\|u_{2}\right\|<\eta \text { and }\left\|u_{2}\right\|_{L^{p}\left(\mathbb{R}^{N}\right)}<\eta \text { for all } m \geq m^{*} .
$$

From (4.32) and (4.33) we get

$$
\frac{d}{d t}\left(\left\|u_{2}\right\|^{2}+\varepsilon\left\|\nabla u_{2}\right\|^{2}\right)+\delta\left(\left\|u_{2}\right\|^{2}+\varepsilon\left\|\nabla u_{2}\right\|^{2}\right) \leq C \eta+\|\sigma\|_{\infty} \eta,
$$

thus

$$
\left\|u_{2}\right\|^{2}+\varepsilon\left\|\nabla u_{2}\right\|^{2} \leq e^{-\delta(t-T)}\left(\|u(T)\|^{2}+\varepsilon\|\nabla u(T)\|^{2}\right)+C \eta .
$$

Thus, we can find $t^{*} \geq \tau, m_{0} \in \mathbb{N}$, such that

$$
\left\|u_{2}\right\|^{2}+\varepsilon\left\|\nabla u_{2}\right\|^{2} \leq C \eta
$$

for any $t \geq t^{*}, u_{\tau} \in B$ and $m \geq m_{0}$. This shows that the process $\left\{U_{\sigma}(t, \tau)\right\}_{\sigma \in \Sigma}$ satisfies condition (ii) in Lemma 4.9. The condition (i) is obviously satisfied since $\bigcup_{\sigma \in \Sigma} \bigcup_{t \geq t^{*}} U_{\sigma}(t, \tau) B$ is bounded and $P_{m}$ is a bounded projector for any $m$. Then, by Lemma 4.9 , we see that $\left\{U_{\sigma}(t, \tau)\right\}_{\sigma \in \mathcal{H}_{w}(g)}$ is $\left(H^{1}\left(\mathbb{R}^{N}\right) \cap\right.$ $L^{p}\left(\mathbb{R}^{N}\right), H^{1}\left(\mathbb{R}^{N}\right)$ )-uniformly asymptotically compact. Combining this with the existence of the $\left(H^{1}\left(\mathbb{R}^{N}\right) \cap L^{p}\left(\mathbb{R}^{N}\right), L^{p}\left(\mathbb{R}^{N}\right)\right)$-uniform attractor, we obtain the existence of an $\left(H^{1}\left(\mathbb{R}^{N}\right) \cap L^{p}\left(\mathbb{R}^{N}\right), H^{1}\left(\mathbb{R}^{N}\right) \cap L^{p}\left(\mathbb{R}^{N}\right)\right)$-uniform attractor $\mathcal{A}_{\mathcal{H}_{w}(g)}$. The structure of $\mathcal{A}_{\mathcal{H}_{w}(g)}$ follows directly from Theorem 2.1.

\section{The upper semicontinuity of uniforms attractors at $\varepsilon=0$}

Hereafter, we denote by $\left\{U_{\sigma}^{\varepsilon}(t, \tau)\right\}$ the process associated to equation (1.1) with $-\varepsilon \Delta u_{t}$ term and the external force $\sigma$.

Lemma 5.1. Assume that $\left\{\phi_{n}\right\}$ is a bounded sequence in $H^{1}\left(\mathbb{R}^{N}\right) \cap L^{p}\left(\mathbb{R}^{N}\right)$, $\left\{\sigma_{n}\right\} \subset \mathcal{H}_{w}(g)$, and $\left\{\varepsilon_{n}\right\} \subset[0,1]$ satisfying

$$
\begin{gathered}
\phi_{n} \rightarrow \phi \text { in } H^{1}\left(\mathbb{R}^{N}\right) \cap L^{p}\left(\mathbb{R}^{N}\right), \\
\sigma_{n} \rightarrow \sigma \text { in } \mathcal{H}_{w}(g),
\end{gathered}
$$

and

$$
\varepsilon_{n} \rightarrow 0
$$

as $n \rightarrow+\infty$. Then for all $t \geq \tau$, there exists a subsequence $\{j\}$ of $\{n\}$ such that

$$
U_{\sigma_{j}}^{\varepsilon_{j}}(t, \tau) \phi_{j} \rightarrow U_{\sigma}^{0}(t, \tau) \phi \text { in } L^{2}\left(\mathbb{R}^{N}\right)
$$


Proof. Denoting $u_{n}(t)=U_{\sigma_{n}}(t, \tau) \phi_{n}$, we get

$$
\partial_{t} u_{n}-\varepsilon_{n} \Delta \partial_{t} u_{n}-\Delta u_{n}+f\left(x, u_{n}\right)+\lambda u_{n}=\sigma_{n}(t) .
$$

Multiplying (5.4) by $u_{n}+\partial_{t} u_{n}$, integrating over $\mathbb{R}^{N}$, and applying the Hölder and Cauchy inequalities, we obtain

$$
\begin{aligned}
& \frac{d}{d t}\left((\lambda+1)\left\|u_{n}\right\|^{2}+\left(\varepsilon_{n}+1\right)\left\|\nabla u_{n}\right\|^{2}+2 \int_{\mathbb{R}^{N}} F\left(x, u_{n}\right)\right) \\
& +2\left\|\nabla u_{n}\right\|^{2}+2 \lambda\left\|u_{n}\right\|^{2}+\left\|\partial_{t} u_{n}\right\|^{2}+2 \varepsilon\left\|\nabla \partial_{t} u_{n}\right\|^{2}+2 \int_{\mathbb{R}^{N}} f\left(x, u_{n}\right) u_{n} \\
\leq & \left(1+\frac{1}{\lambda}\right)\left\|\sigma_{n}(t)\right\|^{2} .
\end{aligned}
$$

Integrating (5.5) from $\tau$ to $t$, using (1.2), (1.5) and noting that $u_{n}(\tau)=\phi_{n}$, we have

$$
\begin{aligned}
& (\lambda+1)\left\|u_{n}(t)\right\|^{2}+\left\|\nabla u_{n}(t)\right\|^{2}+\alpha_{3}\left\|u_{n}(t)\right\|_{L^{p}\left(\mathbb{R}^{N}\right)}^{p} \\
& +2 \int_{\tau}^{t}\left\|\nabla u_{n}\right\|^{2}+\lambda \int_{\tau}^{t}\left\|u_{n}\right\|^{2}+2 \varepsilon_{n} \int_{\tau}^{t}\left\|\nabla \partial_{t} u_{n}\right\|^{2} \\
\leq & (\lambda+1)\left\|\phi_{n}\right\|^{2}+2\left\|\nabla \phi_{n}\right\|^{2}+2 \alpha_{4}\left\|\phi_{n}\right\|_{L^{p}\left(\mathbb{R}^{N}\right)}^{p}+2\left\|\beta_{4}\right\|_{L^{1}\left(\mathbb{R}^{N}\right)} \\
& +2(t-\tau)\left\|\beta_{1}\right\|_{L^{1}\left(\mathbb{R}^{N}\right)}+\left(1+\frac{1}{\lambda}\right) \int_{\tau}^{t}\left\|\sigma_{n}(s)\right\|^{2} .
\end{aligned}
$$

By (5.1) and (5.2) we deduce that the right-hand side of (5.6) is bounded by a constant $C$ independent of $n$. Thus, from (5.6) we see that

$$
\left\{u_{n}(t)\right\} \text { is bounded in } H^{1}\left(\mathbb{R}^{N}\right) \cap L^{p}\left(\mathbb{R}^{N}\right) .
$$

Thus, there exists a function $v_{0} \in L^{2}\left(\mathbb{R}^{N}\right)$ such that $u_{n}(t) \rightarrow v_{0}$ weakly in $L^{2}\left(\mathbb{R}^{N}\right)$ (up to a subsequence). For each $m>0$, we denote by $B_{m}$ the ball centered at origin with radius $m$. Take any $\psi \in L^{2}\left(B_{m}\right)$, we set $\bar{\psi}(x)=\psi(x)$ for all $x \in B_{m}$ and $\bar{\psi}(x)=0$ for all $x>m$. It is obvious that $\bar{\psi} \in L^{2}\left(\mathbb{R}^{N}\right)$ and

$$
\left(u_{n}(t), \psi\right)_{L^{2}\left(B_{m}\right)}=\left(u_{n}(t), \bar{\psi}\right)_{L^{2}\left(\mathbb{R}^{N}\right)} \rightarrow\left(v_{0}, \bar{\psi}\right)_{L^{2}\left(\mathbb{R}^{N}\right)}=\left(v_{0}, \psi\right)_{L^{2}\left(B_{m}\right)} .
$$

It implies that $u_{n}(t) \rightarrow v_{0}$ in $L^{2}\left(B_{m}\right)$ for all $m>0$. On the other hand, for $m>0,\left\{u_{n}(t)\right\}$ is bounded in $H^{1}\left(B_{m}\right)$, then since $H^{1}\left(B_{m}\right) \hookrightarrow L^{2}\left(B_{m}\right)$ compactly, we find that $\left\{u_{n}(t)\right\}$ is precompact in $L^{2}\left(B_{m}\right)$. By a diagonal procedure, we can choose a subsequence $\{j\}$ of $\{n\}$ such that $u_{j}(t) \rightarrow v_{m}$ strongly in $L^{2}\left(B_{m}\right)$, where $v_{m} \in L^{2}\left(B_{m}\right)$. By the uniqueness of the weak limit we conclude that

$$
u_{j}(t) \rightarrow v_{0} \text { strongly in } L^{2}\left(B_{m}\right) \text { for all } m>0 .
$$

We will prove that $u_{j}(t) \rightarrow v_{0}$ in $L^{2}\left(\mathbb{R}^{N}\right)$. Indeed, we have

$$
\int_{\mathbb{R}^{N}}\left|u_{j}(t)-v_{0}\right|^{2} \leq \int_{B_{m}}\left|u_{j}(t)-v_{0}\right|^{2}+2 \int_{B_{m}^{c}}\left|u_{j}(t)\right|^{2}+2 \int_{B_{m}^{c}}\left|v_{0}\right|^{2},
$$


where $B_{m}^{c}=\left\{x \in \mathbb{R}^{N}:|x|>m\right\}$. We now control terms of the right-hand side of (5.8). First, by (5.7) we get

$$
\int_{B_{m}}\left|u_{j}(t)-v_{0}\right|^{2} \rightarrow 0 \text { as } n \rightarrow+\infty
$$

Next, using arguments in Lemma 4.3, we easily deduce that

$$
\begin{aligned}
\int_{B_{m}^{c}}\left|u_{j}(t)\right|^{2} \leq & e^{-\delta(t-\tau)} \int_{B_{m}^{c}}\left(\left|\phi_{j}\right|^{2}+\left|\nabla \phi_{j}\right|^{2}\right)+C \int_{\tau}^{t} \int_{B_{m}^{c}}\left|\sigma_{j}(s)\right|^{2} \\
& +C \int_{B_{m}^{c}}\left|\beta_{1}(x)\right|+\frac{C}{m} \int_{\tau}^{t}\left(\left\|u_{j}(s)\right\|^{2}+\left\|\nabla u_{j}(s)\right\|^{2}+\varepsilon_{j}^{2}\left\|\nabla \partial_{t} u_{j}\right\|^{2}\right) .
\end{aligned}
$$

Applying (5.1), (5.2) and (5.6) in (5.9) gives us

$$
\int_{B_{m}^{c}}\left|u_{j}(t)\right|^{2} \rightarrow 0 \text { as } n, m \rightarrow+\infty .
$$

Because $v_{0} \in L^{2}\left(\mathbb{R}^{N}\right)$,

$$
\int_{B_{m}^{c}}\left|v_{0}\right|^{2} \rightarrow 0 \text { as } m \rightarrow+\infty .
$$

Combining (5.8)-(5.11), we claim that

$$
u_{j}(t) \rightarrow v_{0} \text { in } L^{2}\left(\mathbb{R}^{N}\right) \text { as } n \rightarrow+\infty .
$$

On the other hand, doing similarly to Lemma 3.2, we have

$$
U_{\sigma_{j}}^{\varepsilon_{j}}(t, \tau) \phi_{j} \rightarrow U_{\sigma}^{0}(t, \tau) \phi \text { in } H^{1}\left(\mathbb{R}^{N}\right) \cap L^{p}\left(\mathbb{R}^{N}\right) .
$$

From (5.12) and (5.13) we get (5.3).

Theorem 5.2. Assume that $f$ satisfies (H1) and $g$ satisfies (H2"). Then the family of uniform attractors $\left\{\mathcal{A}_{\varepsilon}\right\}_{\varepsilon \in[0,1]}$ is upper semicontinuous in $L^{2}\left(\mathbb{R}^{N}\right)$ at $\varepsilon=0$, that is,

$$
\lim _{\varepsilon \rightarrow 0} \operatorname{dist}_{L^{2}\left(\mathbb{R}^{N}\right)}\left(\mathcal{A}_{\varepsilon}, \mathcal{A}_{0}\right)=0 .
$$

Proof. Assume that $\operatorname{dist}_{L^{2}\left(\mathbb{R}^{N}\right)}\left(\mathcal{A}_{\varepsilon}, \mathcal{A}_{0}\right) \not \rightarrow 0$ as $\varepsilon \rightarrow 0$. Then there exists $\delta>0$ such that

$$
\limsup _{\varepsilon \rightarrow 0} \operatorname{dist}_{L^{2}\left(\mathbb{R}^{N}\right)}\left(\mathcal{A}_{\varepsilon}, \mathcal{A}_{0}\right) \geq \delta .
$$

Since $\mathcal{A}_{\varepsilon}$ is compact for any $\varepsilon \in[0,1]$, we can choose a sequence $\varepsilon_{n}, \varepsilon_{n} \rightarrow 0$ as $n \rightarrow+\infty$ and $\psi_{n} \in \mathcal{A}_{\varepsilon_{n}}$ satisfying

$$
\operatorname{dist}_{L^{2}\left(\mathbb{R}^{N}\right)}\left(\psi_{n}, \mathcal{A}_{0}\right) \geq \delta \text { for all } n \geq 1 \text {. }
$$

By Proposition 4.1 and Theorem 2.1 we see that the set $\mathfrak{A}=\bigcup_{\varepsilon \in(0 ; 1]} \mathcal{A}_{\varepsilon}$ is bounded, and then by the uniform attracting property of $\mathcal{A}_{0}$, we can choose $t$ large enough such that

$$
\operatorname{dist}_{L^{2}\left(\mathbb{R}^{N}\right)}\left(U_{\sigma}^{0}(t, 0) \mathfrak{A}, \mathcal{A}_{0}\right) \leq \frac{\delta}{2} \text { for all } \sigma \in \mathcal{H}_{w}(g) .
$$


From Theorem 4.4, we know that

$$
\mathcal{A}_{\varepsilon_{n}}=\bigcup_{\sigma \in \mathcal{H}_{w}(g)} \mathcal{K}_{\sigma}^{\varepsilon_{n}}(t),
$$

thus, since $\psi_{n} \in \mathcal{A}_{\varepsilon_{n}}$, there exists a $\sigma_{n} \in \mathcal{H}_{w}(g)$ such that $\psi_{n} \in \mathcal{K}_{\sigma_{n}}^{\varepsilon_{n}}(t)$. By definition of $\mathcal{K}_{\sigma_{n}}^{\varepsilon_{n}}$, we get a $\phi_{n} \in \mathcal{K}_{\sigma_{n}}^{\varepsilon_{n}}(0)$ satisfying $\psi_{n}=U_{\sigma_{n}}^{\varepsilon_{n}}(t, 0) \phi_{n}$. Since $\left\{\phi_{n}\right\} \subset \bigcup_{n>1} \mathcal{K}_{\sigma_{n}}^{\varepsilon_{n}}(0)$ is bounded in $H^{1}\left(\mathbb{R}^{N}\right) \cap L^{p}\left(\mathbb{R}^{N}\right), \mathcal{H}_{w}(g)$ is weakly compact and $\lim _{n \rightarrow+\infty} \varepsilon_{n}=0$ we obtain a subsequence $\{m\} \subset\{n\}$ such that

$$
\begin{gathered}
\phi_{m} \rightarrow \phi \text { in } H^{1}\left(\mathbb{R}^{N}\right) \cap L^{p}\left(\mathbb{R}^{N}\right), \\
\sigma_{m} \rightarrow \sigma_{0} \text { in } L^{2}\left(\tau, t ; L^{2}\left(\mathbb{R}^{N}\right)\right) \text {, and } \\
\varepsilon_{m} \rightarrow 0
\end{gathered}
$$

as $m \rightarrow+\infty$. Now applying Lemma 5.1, we deduce that

$$
\psi_{m} \rightarrow U_{\sigma_{0}}^{0}(t, 0) \phi \in U_{\sigma_{0}}^{0}(t, 0) \mathfrak{A},
$$

which contradicts (5.14) and (5.15). This completes the proof.

\section{Relationships between pullback attractors, uniform attractors and global attractors}

In this section we discuss relationships between the above uniform attractor, the pullback attractor obtained in [3], and the global attractor obtained when the external force $f$ does not depend on the time variable $t$.

\subsection{A relationship between uniform attractors and global attractors}

Let us now briefly consider the matter of the existence of a global attractor when the function $f \in L^{2}\left(\mathbb{R}^{N}\right)$ does not depend on the time variable $t$, i.e., in the autonomous case.

In this case we can define a continuous semigroup $S(t): H^{1}\left(\mathbb{R}^{N}\right) \cap L^{p}\left(\mathbb{R}^{N}\right) \rightarrow$ $H^{1}\left(\mathbb{R}^{N}\right) \cap L^{p}\left(\mathbb{R}^{N}\right)$ by

$$
S(t) u_{0}=u(t)
$$

where $u(t)$ is the unique weak solution to problem (1.1) corresponding to the initial datum $u_{0}$. It is easy to see that

$$
S(t) u_{0}=U(t, 0) u_{0}=U(t+\tau, \tau) u_{0} \text { for any } \tau \in \mathbb{R} .
$$

We recall that a compact set $\mathcal{A}$ is said to be a global attractor for $S(t)$ if it is invariant (i.e., $S(t) \mathcal{A}=\mathcal{A}, \forall t \geq 0$ ) and attracts every bounded subset $B$ of $H^{1}\left(\mathbb{R}^{N}\right) \cap L^{p}\left(\mathbb{R}^{N}\right)$, i.e.,

$$
\operatorname{dist}(S(t) B, \mathcal{A}) \rightarrow 0 \text { as } t \rightarrow+\infty .
$$

From estimate (4.5), we see that the set

$$
B_{0}=\left\{u \in H^{1}\left(\mathbb{R}^{N}\right) \cap L^{p}\left(\mathbb{R}^{N}\right):\|u\|^{2}+\|\nabla u\|^{2}+\|u\|_{p}^{p} \leq \rho_{0}\right\}
$$

is a bounded absorbing set for $S(t)$, i.e., for any bounded subset $B$ there is $T(B)$ such that $S(t) B \subset B_{0}$ as soon as $t \geq T(B)$. 
On the other hand, for any $t_{n} \rightarrow+\infty$ and $u_{n} \in B$, the sequence

$$
S\left(t_{n}\right) u_{n}=U\left(t_{n}, 0\right) u_{n}
$$

is relatively compact in $H^{1}\left(\mathbb{R}^{N}\right) \cap L^{p}\left(\mathbb{R}^{N}\right)$ due to Lemmas 4.9 and 4.10 . Hence, $S(t)$ is asymptotically compact.

Then, it follows from standard theorems (see, e.g., [5]) that the semigroup $S(t)$ posesses a connected compact global attractor $\mathcal{A}$ in $H^{1}\left(\mathbb{R}^{N}\right) \cap L^{p}\left(\mathbb{R}^{N}\right)$.

Thus, in the autonomous case, our results are even new and extend some results for the nonclassical diffusion equation in bounded domains [10] to the case of unbounded domains.

\subsection{A relationship between pullback attractors and uniform attrac- tors}

Let now the external force $g$ satisfy condition (H2"). By Theorem 3.12 in [3], it deduces that for any $\sigma \in \mathcal{H}_{w}(g)$, the process $\left\{U_{\sigma}(t, \tau)\right\}$ has a pullback attractor $\hat{\mathcal{A}}_{\sigma}=\left\{A_{\sigma}(t): t \in \mathbb{R}\right\}$ in $H^{1}\left(\mathbb{R}^{N}\right) \cap L^{p}\left(\mathbb{R}^{N}\right)$. We now prove the following

Theorem 6.1. Assume conditions (H1) and (H2") hold. Then for any $\sigma \in$ $\mathcal{H}_{w}(g)$, the process $U_{\sigma}(t, \tau)$ has a pullback attractor $\hat{\mathcal{A}}_{\sigma}=\left\{A_{\sigma}(t): t \in \mathbb{R}\right\}$ in $H^{1}\left(\mathbb{R}^{N}\right) \cap L^{p}\left(\mathbb{R}^{N}\right)$, and

$$
A_{\sigma}(s)=\mathcal{K}_{\sigma}(s), \quad \bigcup_{\sigma \in \mathcal{H}_{w}(g)} A_{\sigma}(s)=\mathcal{A}_{\mathcal{H}_{w}(g)}, \quad \forall s \in \mathbb{R},
$$

where $\mathcal{A}_{\mathcal{H}_{w}(g)}$ is the uniform attractor of problem $(1.1), \mathcal{K}_{\sigma}$ is the kernel of the process $U_{\sigma}(t, \tau)$.

Proof. Since $\hat{\mathcal{A}}_{\sigma}$ is pullback attracting and since $A_{\sigma}(s)$ is compact, we have

$$
\mathcal{K}_{\sigma}(s) \subset A_{\sigma}(s) \text { for any } s \in \mathbb{R} \text {. }
$$

On the other hand, by the definition of $\mathcal{K}_{\sigma}(s)$ and the invariance of $\hat{\mathcal{A}}_{\sigma}$, we have

$$
A_{\sigma}(s) \subset \mathcal{K}_{\sigma}(s) \text { for any } s \in \mathbb{R} \text {. }
$$

So, we have

$$
A_{\sigma}(s)=\mathcal{K}_{\sigma}(s) \text { for any } s \in \mathbb{R} .
$$

Next, by (6.1) and Theorem 4.11,

$$
\mathcal{A}_{\mathcal{H}_{w}(g)}=\bigcup_{\sigma \in \mathcal{H}_{w}(g)} \mathcal{K}_{\sigma}(s)=\bigcup_{\sigma \in \mathcal{H}_{w}(g)} A_{\sigma}(s), \quad \forall s \in \mathbb{R} .
$$

The proof is complete.

Acknowledgements. The authors would like to thank the reviewer for the valuable suggestions which improved the presentation of the paper. 


\section{References}

[1] E. C. Aifantis, On the problem of diffusion in solids, Acta Mech. 37 (1980), no. 3-4, $265-296$.

[2] C. T. Anh and T. Q. Bao, Pullback attractors for a class of non-autonomous nonclassical diffusion equations, Nonlinear Anal. 73 (2010), no. 2, 399-412.

[3] Dynamics of non-autonomous nonclassical diffusion equations on $\mathbb{R}^{N}$, Commun. Pure Appl. Anal. 11 (2012), no. 3, 1231-1252.

[4] G. Chen and C. K. Zhong, Uniform attractors for non-autonomous p-Laplacian equation, Nonlinear Anal. 68 (2008), no. 11, 3349-3363.

[5] V. V. Chepyzhov and M. I. Vishik, Attractors for Equations of Mathematical Physics, Amer. Math. Soc. Colloq. Publ., Vol. 49, Amer. Math. Soc., Providence, RI, 2002.

[6] J.-L. Lions, Quelques Méthodes de Résolution des Problèmes aux Limites Non Linéaires, Dunod, Paris, 1969.

[7] J. C. Peter and M. E. Gurtin, On a theory of heat conduction involving two temperatures, Z. Angew. Math. Phys. 19 (1968), no. 4, 614-627.

[8] H. Song, S. Ma, and C. K. Zhong, Attractors of non-autonomous reaction-diffusion equations, Nonlinearity 22 (2009), no. 3, 667-681.

[9] H. Song and C. K. Zhong, Attractors of non-autonomous reaction-diffusion equations in $L^{p}$, Nonlinear Anal. 68 (2008), no. 7, 1890-1897.

[10] C. Sun, S. Wang, and C. K. Zhong, Global attractors for a nonclassical diffusion equation, Acta Math. Appl. Sin. Engl. Ser. 23 (2007), no. 7, 1271-1280.

[11] C. Sun and M. Yang, Dynamics of the nonclassical diffusion equations, Asymp. Anal. 59 (2008), no. 1-2, 51-81.

[12] R. Temam, Navier-Stokes Equations and Nonlinear Functional Analysis, 2nd edition, Philadelphia, 1995.

[13] T. W. Ting, Certain non-steady flows of second-order fluids, Arch. Ration. Mech. Anal. 14 (1963), 1-26.

[14] C. Truesdell and W. Noll, The Nonlinear Field Theories of Mechanics, Encyclomedia of Physics, Springer, Berlin, 1995.

[15] B. Wang, Attractors for reaction-diffusion equations in unbounded domains, Phys. D 179 (1999), no. 1, 41-52.

[16] S. Wang, D. Li, and C. K. Zhong, On the dynamic of a class of nonclassical parabolic equations, J. Math. Anal. Appl. 317 (2006), no. 2, 565-582.

[17] H. Wu and Z. Zhang, Asymptotic regularity for the nonclassical diffusion equation with lower regular forcing term, Dyn. Syst. 26 (2011), no. 4, 391-400.

[18] Y. Xiao, Attractors for a nonclassical diffusion equation, Acta Math. Appl. Sin. Engl. Ser. 18 (2002), no. 2, 273-276.

Cung The Anh

Department of Mathematics

Hanoi National University of Education

136 Xuan Thuy, Cau Giay, Hanoi, Vietnam

E-mail address: anhctmath@hnue.edu.vn

NGuYen DuOng TOAN

Department of Mathematics

HAIPHONG UNIVERSITY

171 Phan Dang Luu, Kien An, Haiphong, Vietnam

E-mail address: ngduongtoanhp@gmail.com 